\title{
Continuous Recursive Sliding Mode Control for Hypersonic Flight Vehicle with Extended Disturbance Observer
}

\author{
Yunjie Wu and Jianmin Wang \\ School of Automation Science and Electrical Engineering, Beihang University, Beijing 100191, China \\ Correspondence should be addressed to Jianmin Wang; wjm123121@126.com
}

Received 9 August 2015; Accepted 20 October 2015

Academic Editor: Ricardo Aguilar-López

Copyright ( 2015 Y. Wu and J. Wang. This is an open access article distributed under the Creative Commons Attribution License, which permits unrestricted use, distribution, and reproduction in any medium, provided the original work is properly cited.

\begin{abstract}
A continuous recursive sliding mode controller (CRSMC) with extended disturbance observer (EDO) is proposed for the longitudinal dynamics of a generic hypersonic flight vehicle (HFV) in the presence of multiple uncertainties under control constraints. Firstly, sliding mode tracking controller based on a set of novel recursive sliding mode manifolds is presented, in which the chattering problem is reduced. The CRSMC possesses the merits of both nonsingular terminal sliding mode controller (NTSMC) and high-order sliding mode controller (HOSMC). Then antiwindup controller is designed according to the input constraints, which adds a dynamic compensation factor in the CRSMC. For the external disturbance of system, an improved disturbance observer based on extended disturbance observer (EDO) is designed. The external disturbance is estimated by the disturbance observer and the estimated value is regarded as compensation in CRSMC for disturbance. The stability of the proposed scheme is analyzed by Lyapunov function theory. Finally, numerical simulation is conducted for cruise flight dynamics of HFV, where altitude is $110000 \mathrm{ft}$, velocity is $15060 \mathrm{ft} / \mathrm{s}$, and Mach is 15 . Simulation results show the validity of the proposed approach.
\end{abstract}

\section{Introduction}

Hypersonic flight vehicle (HFV) is a cost-effective and reliable space aircraft. It can realize prompt global responses for its high speed. So HFV has attracted more and more attention from the civil and military field. However, HFV is highly coupling and nonlinear aircraft, and it is extremely sensitive to changes in physical and aerodynamic parameters due to its peculiar structure and rigorous flight environment. Hence, it is a significantly challenging task to model and control HFV. Since the 1960s, a large number of scholars had done research in this field. Up to now, abundant research results have been achieved.

The aeropropulsive and aeroelastic properties of HFV were analyzed in detail by Chavez and Schmidt [1], which laid the foundation for establishing HFV model. Bolender and Doman built a nonlinear longitudinal dynamics model for the air-breathing HFV in $[2,3]$, which captured the complex interactions between the aerodynamics, propulsion system, and structural dynamics. Then, robust flight control systems based on Monte Carlo evaluation were synthesized in [4], and stochastic robust nonlinear dynamics inversion (NDI) control law was presented for longitudinal motion of HFV in [5]. For the sake of applying linear control method, control-oriented linearized model was derived in [6]. Obaid proposed minimax linear quadratic regulator (LQR) optimal controller in $[7,8]$ and minimax optimal linear quadratic Gaussian controller in [9] based on feedback linearization model of HFV by virtue of minimax linear quadratic theory, where the optimal control minimizes the maximum value of the quadratic cost function and gives an optimal solution for the selected cost function. The optimal controller can achieve good control performance; however, the construction of Riccati equation parameter matrixes in LQR and LQG is a difficult work. Qi et al. [10] designed an adaptive backstepping fault-tolerant control scheme for HFV by means of backstepping theory. Butt et al. [11] designed adaptive controller for HFV in terms of dynamic surface method. Xu et al. [12] proposed adaptive sliding mode control for longitudinal dynamics of input-output linearization model of HFV, in which the uncertain parameters were estimated by adaptive laws based on Lyapunov synthesis approach. Among all the 
above methods, sliding mode control is the most attractive method, because of its robustness to uncertainty, and it is simple and designed easily.

Terminal sliding mode control is a kind of finite time convergence control, which appeals to many researchers due to its controllable convergence time. Until now, there are numerous research results about terminal sliding mode control, such as continuous terminal sliding mode control design [13] and fast terminal sliding mode control [14]. Nevertheless, terminal sliding mode control usually has singularity problem, which reduces its performance. According to this, Feng et al. [15] proposed nonsingular terminal sliding mode control and applied it to the rigid manipulators successfully.

It is worth noting that HFV is a high-order nonlinear system. Hence, the low-order and linear sliding mode control is conservative and cannot acquire better performance. References [16, 17] have presented high-order sliding mode control for HFV, but their first sliding surfaces remain in a linear form. And the chattering problem of sliding mode control affects the engineering application. In addition, the operation of mechanical actuators for HFV is restricted, which is easy to reach saturation in the near space where the execution efficiency of actuators drops. According to the input saturation, a large number of research results can be consulted, such as [18-20]. While the articles about antiwindup control for HFV are few, only [21] takes into account actuator restraints under complex flight conditions. Aiming at all the above, a continuous recursive sliding mode control (CRSMC) under control constraints is proposed for $\mathrm{HFV}$ in this paper, in which a compensation factor is added in CRSMC for actuator restraints.

Extended state observer (ESO) was proposed by Han in [22]. In ESO, the disturbance is regarded as a system state and then state observer is constructed. Through tuning the observer gain, which is the only adjustable parameter, all states of the system can be estimated. At the same time, the disturbance is estimated as the extended state. ESO is a simple and effective observer, which has been applied in many projects successfully, such as speed control of induction motor drive [23], attitude tracking of rigid spacecraft [24], robotic uncalibrated hand-eye coordination control [25], and boiler-turbine-generator control systems [26]. In addition, Gao developed ESO by proposing scaling and bandwidthparameterization theory [27], which simplifies and facilitates the parameter tuning process. Ginoya et al. [28] proposed an extended disturbance observer (EDO) on the basis of disturbance observer (DO), which was projected by Chen in [29-31]. The EDO is appropriate for the system having disturbances in all channels. In accordance with the theory of ESO and EDO, an improved disturbance observer (IEDO) is designed for the external disturbance of HFV in this paper. The external disturbance is estimated by IEDO. Then, the estimated value is regarded as compensation for the above CRSMC. Therefore, the proposed scheme of this paper is CRSMC under control constraints plus IEDO. The major contributions of this paper are as follows:

(I) A novel recursive sliding mode control is applied in the flight control of HFV, in which the recursive sliding manifolds are nonlinear and nonsingular. In addition, this sliding mode controller is continuous due to its nonlinear reaching law. Compared with the linear sliding mode control for HFV in [12, 16, 17], the proposed scheme has faster response and is less conservative.

(II) A compensation controller for control constraints is proposed for HFV, for which the articles are few. The compensation controller works after the CRSMC as a compensation factor, which not only plays a role in antiwindup controller but also has no effect on the performance of the CRSMC. The simulations in following part demonstrate its effectiveness.

(III) A novel disturbance observer is designed for the external disturbance for HFV. The disturbance observer is improved extended disturbance observer (EDO) in [28], in which there are more parameters that need to be regulated because of each channel with multiple gain parameters. The improved EDO has only one parameter that needs to be regulated, which is more convenient for engineering application.

The remainder of this paper is organized as follows. Section 2 formulates the HFV longitudinal model and control problem. Section 3 reveals the design process of CRSMC under control constraints and IEDO for external disturbance in detail. The stability of the proposed scheme is analyzed in Section 4 by Lyapunov function method. Numerical simulations are conducted in Section 5. Section 6 provides conclusions of the paper.

\section{Model and Problem Formulation}

2.1. HFV Model. The control plant of this paper is a model of longitudinal dynamics of a generic HFV, which is developed by NASA Langley research center. The model consists of some differential equations that describe velocity, altitude, flight path angle, angle of attack, and pitch rate $[12,18]$, which are expressed as follows:

$$
\begin{aligned}
& \dot{V}=\frac{T \cos \alpha-D}{m}-\frac{\mu \sin \gamma}{r^{2}}, \\
& \dot{h}=V \sin \gamma, \\
& \dot{\gamma}=\frac{L+T \sin \alpha}{m V}-\frac{\left(\mu-V^{2} r\right) \cos \gamma}{V r^{2}}, \\
& \dot{\alpha}=q-\dot{\gamma}, \\
& \dot{q}=\frac{M_{y y}}{I_{y y}},
\end{aligned}
$$

where $V, h, \gamma, \alpha, q$ are velocity, altitude, flight path angle, angle of attack, and pitch rate of HFV, respectively; $I_{y y}$ is moment of inertia of the aircraft and $m$ is mass; $L, D, T$, and $M_{y y}$ are, respectively, lift, drag, thrust, and pitching moment acting on the aircraft; $r$ is radial distance from Earth's center. 
The detailed expressions of $L, D, T$, and $M_{y y}$, and $r$ are, respectively, as follows:

$$
\begin{aligned}
L & =\frac{1}{2} \rho V^{2} S C_{L}, \\
D & =\frac{1}{2} \rho V^{2} S C_{D}, \\
T & =\frac{1}{2} \rho V^{2} S C_{T}, \\
M_{y y} & =\frac{1}{2} \rho V^{2} S \bar{c}\left[C_{M}(\alpha)+C_{M}\left(\delta_{e}\right)+C_{M}(q)\right], \\
r & =h+R_{E},
\end{aligned}
$$

where $\rho, S, \bar{c}$, and $R_{E}$ denote, respectively, density of air, reference area, mean aerodynamic chord, and radius of the Earth; $\delta_{e}$ is the elevator deflection angle; $C_{L}, C_{D}, C_{T}$, and $C_{M}(\alpha), C_{M}(q), C_{M}\left(\delta_{e}\right)$ are, respectively, relevant aerodynamic coefficient parameters as

$$
\begin{aligned}
C_{L} & =0.6203 \alpha, \\
C_{D} & =0.6450 \alpha^{2}+0.0043378 \alpha+0.003772, \\
C_{T} & = \begin{cases}0.02576 \beta, & \beta<1 \\
0.0224+0.00336 \beta, & \beta>1,\end{cases} \\
C_{M}(\alpha) & =-0.035 \alpha^{2}+0.036617 \alpha+5.3261 \times 10^{-6}, \\
C_{M}(q) & =\frac{\bar{c} q}{2 V}\left(-6.796 \alpha^{2}+0.3015 \alpha-0.2289\right), \\
C_{M}\left(\delta_{e}\right) & =c_{e}\left(\delta_{e}+d_{2}(t)-\alpha\right),
\end{aligned}
$$

where $d_{2}(t)$ means the external disturbance reflected on the elevator.

The engine dynamics are modeled as a second-order system as

$$
\ddot{\beta}=-2 \xi \omega_{n} \dot{\beta}-\omega_{n}^{2} \beta+\omega_{n}^{2}\left(\beta_{c}+d_{1}(t)\right),
$$

where $\beta$ and $\beta_{c}$ are throttle setting and throttle setting command, respectively; $\xi$ is damping ratio and $\omega_{n}$ is natural frequency of engine; $d_{1}(t)$ is the external disturbance on behalf of torques and generalized elastic forces.

Because of the peculiar structure of HFV and complex flight conditions, some certain parameters uncertainties are taken into consideration; namely,

$$
\begin{aligned}
m & =m_{0}(1+\Delta m), \\
I_{y y} & =I_{0}(1+\Delta I), \\
S & =S_{0}(1+\Delta S), \\
\bar{c} & =\bar{c}_{0}(1+\Delta \bar{c}), \\
\rho & =\rho_{0}(1+\Delta \rho), \\
c_{e} & =c_{e 0}\left(1+\Delta c_{e}\right),
\end{aligned}
$$

where $\left(\cdot_{0}\right)$ represents the nominal value of parameter $(\cdot)$ and $(\Delta \cdot)$ denotes the parameter uncertainties.

Assumption 1. The external disturbance $d_{i}(t)$ is assumed to be upper bounded; that is, $\left|d_{i}(t)\right| \leq g_{i}$; here, $g_{i}$ are known positive constants for $i=1,2$.

Assumption 2. The parameter uncertainties in (5) are assumed to be bounded. And they satisfy the following conditions:

$$
\begin{gathered}
|\Delta m| \leq \Delta m^{*}, \\
|\Delta I| \leq \Delta I^{*} \\
|\Delta S| \leq \Delta S^{*} \\
|\Delta \bar{c}| \leq \Delta \bar{c}^{*} \\
|\Delta \rho| \leq \Delta \rho^{*} \\
\left|\Delta c_{e}\right| \leq \Delta c_{e}^{*}
\end{gathered}
$$

where $\Delta m^{*}, \Delta I^{*}, \Delta S^{*}, \Delta \bar{c}^{*}, \Delta \rho^{*}$, and $\Delta c_{e}^{*}$ are all positive real constants.

Remark 3. According to nonlinear model (1), velocity $V$ and altitude $h$ are regarded as output variables, while the input variables are chosen as engine throttle setting command $\beta_{c}$ and elevator deflection angle $\delta_{e}$. The control task is designing an appropriate controller such that the output variables $(V, h)$ track the relevant command $\left(V_{d}, h_{d}\right)$ in finite time in the presence of disturbance, respectively.

2.2. Input-Output Linearization. Because the model of (1) is highly nonlinear and strong coupling, the linearized model is needed for the sake of designing control law. Here, the input-output linearization method is adopted for linearizing. In accordance with Remark 3 , the linearized target is that the input variables $\left(\beta_{c}, \delta_{e}\right)$ appear apparently in the expressions of output variables $(V, h)$. In terms of Nonlinear System Theory and employing mathematical tools of Lie derivative, the input variables $\left(\beta_{c}, \delta_{e}\right)$ can appear in the motion equations by differentiating $V$ three times and $h$ four times, respectively [12]; namely,

$$
\begin{aligned}
\dot{V}= & f_{V}(\mathbf{x}), \\
\ddot{V}= & \frac{\boldsymbol{\Omega}_{1} \dot{\mathbf{x}}}{m}, \\
\dddot{V}= & \frac{\left(\boldsymbol{\Omega}_{1} \ddot{\mathbf{x}}+\dot{\mathbf{x}}^{T} \boldsymbol{\Omega}_{2} \dot{\mathbf{x}}\right)}{m}, \\
\ddot{h}= & \dot{V} \sin \gamma+V \dot{\gamma} \cos \gamma, \\
\dddot{h}= & \ddot{V} \sin \gamma+2 \dot{V} \dot{\gamma} \cos \gamma-V \dot{\gamma}^{2} \sin \gamma+V \ddot{\gamma} \cos \gamma, \\
h^{(4)}= & \dddot{V} \sin \gamma+3 \ddot{V} \dot{\gamma} \cos \gamma-3 \dot{V}^{2} \sin \gamma+3 \dot{V} \ddot{\gamma} \cos \gamma \\
& -3 V \dot{\gamma} \ddot{\gamma} \sin \gamma-V \dot{\gamma}^{3} \cos \gamma+V \ddot{\gamma} \cos \gamma,
\end{aligned}
$$




$$
\begin{aligned}
& \dot{\gamma}=f_{h}(\mathbf{x}), \\
& \ddot{\gamma}=\Pi_{1} \dot{\mathbf{x}}, \\
& \dddot{\gamma}=\Pi_{1} \ddot{\mathbf{x}}+\dot{\mathbf{x}}^{T} \Pi_{2} \dot{\mathbf{x}},
\end{aligned}
$$

where $\mathbf{x}=\left[\begin{array}{lllll}V & \gamma & \alpha & \beta & h\end{array}\right]^{T}$ is the system state vector and $\boldsymbol{\Omega}_{1}, \boldsymbol{\Omega}_{2}, \boldsymbol{\Pi}_{1}$, and $\boldsymbol{\Pi}_{2}$ are the system equations' firstorder and second-order partial differential to state variables, respectively, whose detailed expressions are exhibited in the Appendix. as

The expressions of $\ddot{\alpha}$ and $\ddot{\beta}$ can be divided into two parts

$$
\begin{aligned}
& \ddot{\alpha}=\ddot{\alpha}_{0}+\frac{\rho V^{2} S \bar{c} c_{e}}{2 I_{y y}}\left(\delta_{e}+d_{2}(t)\right), \\
& \ddot{\beta}=\ddot{\beta}_{0}+\omega_{n}^{2}\left(\beta_{c}+d_{1}(t)\right),
\end{aligned}
$$

where

$$
\begin{aligned}
& \ddot{\alpha}_{0}=\frac{1}{2 I_{y y}} \rho V^{2} S \bar{c}\left[C_{M}(\alpha)+C_{M}(q)-c_{e} \alpha\right]-\ddot{\gamma}, \\
& \ddot{\beta}_{0}=-2 \xi \omega_{n} \dot{\beta}-\omega_{n}^{2} \beta .
\end{aligned}
$$

Given $\ddot{\mathbf{x}}_{0}=\left[\begin{array}{lllll}\ddot{V} & \ddot{\gamma} & \ddot{\alpha}_{0} & \ddot{\beta}_{0} & \ddot{h}\end{array}\right]^{T}$, then

$$
\begin{aligned}
\dddot{V} & =F_{V}+b_{11}\left(\beta_{c}+d_{1}(t)\right)+b_{12}\left(\delta_{e}+d_{2}(t)\right), \\
h^{(4)} & =F_{h}+b_{21}\left(\beta_{c}+d_{1}(t)\right)+b_{22}\left(\delta_{e}+d_{2}(t)\right),
\end{aligned}
$$

where

$$
\begin{array}{rl}
F_{V}= & \frac{\left(\Omega_{1} \ddot{\mathbf{x}}_{0}+\dot{\mathbf{x}}^{T} \boldsymbol{\Omega}_{2} \dot{\mathbf{x}}\right)}{m}, \\
F_{h} & 3 \ddot{V} \dot{\gamma} \cos \gamma-3 \dot{V} \dot{\gamma}^{2} \sin \gamma+3 \dot{V} \ddot{\gamma} \cos \gamma-3 V \dot{\gamma} \ddot{\gamma} \sin \gamma \\
& -V \dot{\gamma}^{3} \cos \gamma+F_{V} \sin \gamma \\
& +V \cos \gamma\left(\Pi_{1} \ddot{\mathbf{x}}_{0}+\dot{\mathbf{x}}^{T} \Pi_{2} \dot{\mathbf{x}}\right), \\
b_{11}= & \left(\frac{\rho V^{2} S c_{\beta} \omega_{n}^{2}}{2 m}\right) \cos \alpha, \\
b_{12}= & -\left(\frac{c_{e} \rho V^{2} S \bar{c}}{2 m I_{y y}}\right)\left(T \sin \alpha+D_{\alpha}\right), \\
b_{21}= & \left(\frac{\rho V^{2} S c_{\beta} \omega_{n}^{2}}{2 m}\right) \sin (\alpha+\gamma), \\
b_{22} & \left(\frac{c_{e} \rho V^{2} S \bar{c}}{2 m I_{y y}}\right)\left[T \cos (\alpha+\gamma)+L_{\alpha} \cos \gamma-D_{\alpha} \sin \gamma\right], \\
= & D_{\alpha}=\frac{\partial D}{\partial \alpha}, L_{\alpha}=\frac{\partial L}{\partial \alpha} .
\end{array}
$$

Then, (10) is rewritten as

$$
\left[\begin{array}{c}
\dddot{V} \\
h^{(4)}
\end{array}\right]=\left[\begin{array}{c}
F_{V} \\
F_{h}
\end{array}\right]+\mathbf{B u}+\mathbf{B}\left[\begin{array}{l}
d_{1}(t) \\
d_{2}(t)
\end{array}\right]
$$

where $\mathbf{B}=\left[\begin{array}{ll}b_{11} & b_{12} \\ b_{21} & b_{22}\end{array}\right], \mathbf{u}=\left[\begin{array}{l}\beta_{c} \\ \delta_{e}\end{array}\right]$.

Assumption 4. Matrix $\mathbf{B}$ is assumed to be invertible.

Remark 5. Matrix B is nonsingular during the entire flight envelope except on a vertical flight path [5] for the inputoutput combination. Hence, Assumption 4 is reasonable.

\section{Controller and Observer Design}

In this section, a continuous recursive sliding mode tracking controller is firstly designed and then a compensation controller is designed for the input saturation. In the third subsection, an improved disturbance observer based on EDO is presented for estimating the external disturbance.

3.1. Tracking Controller Design. Before introducing the tracking controller, two lemmas are presented which will assist in analyzing and proving the theorem.

Lemma 6 (see [13]). Consider the following first-order nonlinear differential equation:

$$
s=\dot{x}+\beta|x|^{\gamma} \operatorname{sgn}(x)=0,
$$

where $x \in R, \beta>0$, and $0<\gamma<1$. Then, the system state will converge to zero in finite time

$$
T=\frac{1}{\beta(1-\gamma)}\left|x_{0}\right|^{1-\gamma},
$$

where $x_{0}$ is the initial condition; that is, $x(0)=x_{0}$.

Lemma 7 (see [32]). Consider the following first-order nonlinear differential equation:

$$
x_{n-k}+\frac{\beta_{n-k} a}{k a-1}\left|\dot{x}_{n-k}\right|^{k-1 / a} \operatorname{sgn}\left(\dot{x}_{n-k}\right)=0 \text {, }
$$

where $x \in R, \beta, a \in R, n, k \in N, 0<k<n, \beta>0$, and $a>1$. Then, the system state will also converge to zero in finite time

$$
\begin{aligned}
T= & \frac{k a-1}{(k-1) a-1}\left(\frac{k a-1}{\beta_{n-k} a}\right)^{-a /(k a-1)} \\
& \cdot\left|x_{0}\right|^{((k-1) a-1) /(k a-1)},
\end{aligned}
$$

where $x_{n-k}(0)=x_{0}$ is the initial condition.

Proof. Due to $\beta>0$ and $a>1$, that is, $\beta a \neq 0$, (15) can be changed as

$$
\dot{x}_{n-k}+\left(\frac{k a-1}{\beta_{n-k} a}\right)^{a /(k a-1)}\left|x_{n-k}\right|^{a /(k a-1)} \operatorname{sgn}\left(x_{n-k}\right)=0 .
$$


In accordance with Lemma 6, the solution of (17) will converge to zero in finite time

$$
T=\frac{1}{\beta^{\prime}\left(1-\gamma^{\prime}\right)}\left|x_{n-k}(0)\right|^{1-\gamma^{\prime}},
$$

where $\beta^{\prime}=\left((k a-1) / \beta_{n-k} a\right)^{a /(k a-1)}$ and $\gamma^{\prime}=a /(k a-1)$, $x_{n-k}(0)=x_{0}$. This completes the proof.

Theorem 8. Consider nonlinear model (1) and linearized model (12) with Assumption 4; the system output variables $V$ and $h$ can track the reference commands $V_{d}$ and $h_{d}$, respectively; meanwhile, they keep stable and robust in the presence of parameter uncertainties, if the controller is selected as

$$
=\mathbf{B}^{-1}\left[\begin{array}{l}
\frac{\left(-k_{11} s_{12}-k_{12}\left|s_{12}\right|^{\rho_{1}} \operatorname{sgn}\left(s_{12}\right)-\Phi_{1}\right)}{\Psi_{1}}+\dddot{V}_{d}-F_{V} \\
\frac{\left(-k_{21} s_{22}-k_{22}\left|s_{22}\right|^{p_{2}} \operatorname{sgn}\left(s_{22}\right)-\Phi_{2}\right)}{\Psi_{2}}+h_{d}^{(4)}-F_{h}
\end{array}\right],
$$

where $\Psi_{1}=\beta_{11}\left|\dot{s}_{11}\right|^{1-1 / a_{1}} \beta_{10}\left|\dot{s}_{10}\right|^{2-1 / a_{1}}, \quad \Psi_{2}=$ $\beta_{21}\left|\dot{s}_{21}\right|^{1-1 / a_{2}} \beta_{20}\left|\dot{s}_{20}\right|^{2-1 / a_{2}}, k_{i j}>0,0<\rho_{i}<1, \beta_{i 0}>0$, $\beta_{i 1}>0, a_{i}>1$ for $i=1,2, j=1,2$, and $s_{i m}(i=1,2$; $m=0,1,2)$ are the recursive sliding mode manifolds which are defined as

$$
\begin{aligned}
& s_{10}=e_{1}, \\
& s_{11}=s_{10}+\beta_{10} \frac{a_{1}}{3 a_{1}-1}\left|\dot{s}_{10}\right|^{3-1 / a_{1}} \operatorname{sgn}\left(\dot{s}_{10}\right), \\
& s_{12}=s_{11}+\beta_{11} \frac{a_{1}}{2 a_{1}-1}\left|\dot{s}_{11}\right|^{2-1 / a_{1}} \operatorname{sgn}\left(\dot{s}_{11}\right), \\
& s_{20}=\dot{e}_{2}+\lambda_{2} e_{2}, \\
& s_{21}=s_{20}+\beta_{20} \frac{a_{2}}{3 a_{2}-1}\left|\dot{s}_{20}\right|^{3-1 / a_{2}} \operatorname{sgn}\left(\dot{s}_{20}\right), \\
& s_{22}=s_{21}+\beta_{21} \frac{a_{2}}{2 a_{2}-1}\left|\dot{s}_{21}\right|^{2-1 / a_{2}} \operatorname{sgn}\left(\dot{s}_{21}\right),
\end{aligned}
$$

where $e_{1}=V-V_{d}$ and $e_{2}=h-h_{d}$ are tracking errors and $\lambda_{2}$ is strictly positive constant.

Proof. Taking into account the above recursive sliding mode manifolds, the time differential of (20a), (20b), and (20c) is that

$$
\begin{aligned}
\dot{s}_{10}= & \dot{e}_{1}, \\
\dot{s}_{11}= & \dot{s}_{10}+\beta_{10}\left|\dot{s}_{10}\right|^{2-1 / a_{2}} \ddot{s}_{10}, \\
\ddot{s}_{11}= & \ddot{e}_{1}+\beta_{10}\left(2-\frac{1}{a_{1}}\right)\left|\dot{e}_{1}\right|^{1-1 / a_{1}}+\beta_{10}\left|\dot{e}_{1}\right|^{2-1 / a_{1}} \dddot{e}_{1}, \\
\dot{s}_{12}= & \dot{s}_{11} \\
& +\beta_{11}\left|\dot{s}_{11}\right|^{1-1 / a_{1}}\left[\ddot{e}_{1}+\beta_{10}\left(2-\frac{1}{a_{1}}\right)\left|\dot{e}_{1}\right|^{1-1 / a_{1}}\right] \\
& +\beta_{11}\left|\dot{s}_{11}\right|^{1-1 / a_{1}} \beta_{10}\left|\dot{s}_{10}\right|^{2-1 / a_{1}} \dddot{e}_{1} .
\end{aligned}
$$

In line with $\dddot{e}_{1}=\ddot{V}-\dddot{V}_{d}$, (23) changes as

$$
\dot{s}_{12}=\Phi_{1}+\Psi_{1}\left(\dddot{V}-\dddot{V}_{d}\right),
$$

where

$$
\begin{aligned}
\Phi_{1}= & \dot{s}_{11} \\
& +\beta_{11}\left|\dot{s}_{11}\right|^{1-1 / a_{1}}\left[\ddot{e}_{1}+\beta_{10}\left(2-\frac{1}{a_{1}}\right)\left|\dot{e}_{1}\right|^{1-1 / a_{1}}\right], \\
\Psi_{1}= & \beta_{11}\left|\dot{s}_{11}\right|^{1-1 / a_{1}} \beta_{10}\left|\dot{s}_{10}\right|^{2-1 / a_{1}} .
\end{aligned}
$$

Similarly, the time differential of (21a), (21b), and (21c) is that

$$
\begin{aligned}
\dot{s}_{20} & =\ddot{e}_{2}+\lambda_{2} \dot{e}_{2}, \\
\ddot{s}_{20} & =\dddot{e}_{2}+\lambda_{2} \ddot{e}_{2}, \\
\dddot{s}_{20} & =e_{2}^{(4)}+\lambda_{2} \ddot{e}_{2}, \\
\dot{s}_{21} & =\dot{s}_{20}+\beta_{20}\left|\dot{s}_{20}\right|^{2-1 / a_{2}} \ddot{s}_{20}, \\
\ddot{s}_{21} & =\ddot{s}_{20}+\beta_{20}\left(2-\frac{1}{a_{2}}\right)\left|\dot{s}_{20}\right|^{1-1 / a_{2}} \ddot{s}_{20}^{2} \\
& +\beta_{20}\left|\dot{s}_{20}\right|^{2-1 / a_{2}} \dddot{s}_{20}, \\
\dot{s}_{22} & =\dot{s}_{21}+\beta_{21}\left|\dot{s}_{21}\right|^{1-1 / a_{2}} \\
& \cdot\left[\ddot{s}_{20}+\beta_{20}\left(2-\frac{1}{a_{2}}\right)\left|\dot{s}_{20}\right|^{1-1 / a_{2}} \ddot{s}_{20}^{2}\right] \\
& +\beta_{21}\left|\dot{s}_{21}\right|^{1-1 / a_{2}} \beta_{20}\left|\dot{s}_{20}\right|^{2-1 / a_{2}} \lambda_{2} \ddot{e}_{2}+\beta_{21}\left|\dot{s}_{21}\right|^{1-1 / a_{2}} \\
& \cdot \beta_{20}\left|\dot{s}_{20}\right|^{2-1 / a_{2}} e_{2}^{(4)} .
\end{aligned}
$$

In terms of $e_{2}^{(4)}=h^{(4)}-h_{d}^{(4)},(28)$ is changed as

$$
\dot{s}_{22}=\Phi_{2}+\Psi_{2}\left(h^{(4)}-h_{d}^{(4)}\right),
$$

where

$$
\begin{aligned}
& \Phi_{2}=\dot{s}_{21}+\beta_{21}\left|\dot{s}_{21}\right|^{1-1 / a_{2}} \\
& \cdot\left[\ddot{s}_{20}+\beta_{20}\left(2-\frac{1}{a_{2}}\right)\left|\dot{s}_{20}\right|^{1-1 / a_{2}} \ddot{s}_{20}^{2}\right]+\Psi_{2} \lambda_{2} \ddot{e}_{2}, \\
& \Psi_{2}=\beta_{21}\left|\dot{s}_{21}\right|^{1-1 / a_{2}} \beta_{20}\left|\dot{s}_{20}\right|^{2-1 / a_{2}} .
\end{aligned}
$$

Like that, (24) and (29) are written together as

$$
\left[\begin{array}{c}
\dot{s}_{12} \\
\dot{s}_{22}
\end{array}\right]=\left[\begin{array}{c}
\Phi_{1} \\
\Phi_{2}
\end{array}\right]+\left[\begin{array}{cc}
\Psi_{1} & 0 \\
0 & \Psi_{2}
\end{array}\right]\left[\begin{array}{c}
\dddot{V}-\ddot{V}_{d} \\
h^{(4)}-h_{d}^{(4)}
\end{array}\right] .
$$

Substituting (12) into (31) in the absence of external disturbance yields

$$
\left[\begin{array}{c}
\dot{s}_{12} \\
\dot{s}_{22}
\end{array}\right]=\left[\begin{array}{c}
\Phi_{1} \\
\Phi_{2}
\end{array}\right]+\left[\begin{array}{cc}
\Psi_{1} & 0 \\
0 & \Psi_{2}
\end{array}\right]\left(\left[\begin{array}{c}
F_{V} \\
F_{h}
\end{array}\right]-\left[\begin{array}{c}
\dddot{V}_{d} \\
h_{d}^{(4)}
\end{array}\right]+\mathbf{B u}\right) .
$$


Choose a Lyapunov function candidate as

$$
L_{a}=\frac{1}{2} s^{T} s
$$

where $s=\left[\begin{array}{ll}s_{12} & s_{22}\end{array}\right]^{T}$.

Taking first-order time derivative of Lyapunov function in (33) yields

$$
\begin{aligned}
& \dot{L}_{a}=s^{T} \dot{s} \\
& \quad=s^{T}\left[\left[\begin{array}{l}
\Phi_{1} \\
\Phi_{2}
\end{array}\right]+\left[\begin{array}{cc}
\Psi_{1} & 0 \\
0 & \Psi_{2}
\end{array}\right]\left(\left[\begin{array}{c}
F_{V} \\
F_{h}
\end{array}\right]-\left[\begin{array}{c}
\dddot{V}_{d} \\
h_{d}^{(4)}
\end{array}\right]+\mathbf{B u}\right)\right] .
\end{aligned}
$$

Substituting (19) into (34), we obtain that

$$
\begin{aligned}
\dot{L}_{a} & =s^{T}\left[\begin{array}{l}
-k_{11} s_{12}-k_{12}\left|s_{12}\right|^{\rho_{1}} \operatorname{sgn}\left(s_{12}\right) \\
-k_{21} s_{22}-k_{22}\left|s_{22}\right|^{\rho_{2}} \operatorname{sgn}\left(s_{22}\right)
\end{array}\right] \\
& =-k_{11} s_{12}^{2}-k_{12}\left|s_{12}\right|^{\rho_{1}+1}-k_{21} s_{22}^{2}-k_{22}\left|s_{22}\right|^{\rho_{2}+1} \\
& \leq 0 .
\end{aligned}
$$

Hence, the sliding mode manifolds $s_{12}$ and $s_{22}$ will converge to zero in finite time; that is, the finite convergence time is $t_{i 1}=\left[\ln \left(k_{i 1} s_{i 2}(0)^{1-\rho_{i}}+k_{i 2}\right)-\ln \left(k_{i 2}\right)\right] / k_{i 1}\left(1-\rho_{i}\right)$ for $i=1,2$. This means that

$$
s_{i 2}=0, \quad \forall t>t_{i 1}, \quad i=1,2 .
$$

Substituting (36) into (20c) and (21c), we obtain

$$
\begin{aligned}
& s_{12}=s_{11}+\beta_{11} \frac{a_{1}}{2 a_{1}-1}\left|\dot{s}_{11}\right|^{2-1 / a_{1}} \operatorname{sgn}\left(\dot{s}_{11}\right)=0, \\
& s_{22}=s_{21}+\beta_{21} \frac{a_{2}}{2 a_{2}-1}\left|\dot{s}_{21}\right|^{2-1 / a_{2}} \operatorname{sgn}\left(\dot{s}_{21}\right)=0 .
\end{aligned}
$$

By virtue of Lemma 7, the secondary sliding mode manifolds $s_{11}$ and $s_{21}$ will converge to zero in finite time

$$
\begin{array}{r}
t_{i 2}=\frac{2 a_{i}-1}{a_{i}-1}\left(\frac{2 a_{i}-1}{\beta_{i 1} a_{i}}\right)^{-a_{i} /\left(2 a_{i}-1\right)}\left|s_{i 1}(0)\right|^{\left(a_{i}-1\right) /\left(2 a_{i}-1\right)}, \\
i=1,2 .
\end{array}
$$

Similar to the above process, the last level sliding mode surfaces $s_{10}$ and $s_{20}$ in (20b) and (21b) will also converge to zero in finite time

$$
\begin{array}{r}
t_{i 3}=\frac{3 a_{i}-1}{2 a_{i}-1}\left(\frac{3 a_{i}-1}{\beta_{i 0} a_{i}}\right)^{-a_{i} /\left(3 a_{i}-1\right)}\left|s_{i 0}(0)\right|^{\left(2 a_{i}-1\right) /\left(3 a_{i}-1\right)}, \\
i=1,2 .
\end{array}
$$

Substituting $s_{10}=0$ and $s_{20}=0$ into (20a) and (21a), respectively, it is obtained that

$$
\begin{aligned}
& s_{10}=e_{1}=0, \\
& s_{20}=\dot{e}_{2}+\lambda_{2} e_{2}=0 .
\end{aligned}
$$

It is seen that velocity tracking error $e_{1}$ converges to zero. However, (41) is asymptotic convergence. Define an arbitrary small neighborhood for $e_{2}$; that is, $Z_{e_{2}} \in[-\delta,+\delta]$, $\delta>0$. Once the altitude tracking error converges to the neighborhood, it is considered that the altitude $h$ has tracked the command $h_{d}$. So the approximate convergence time is

$$
t_{e_{2}} \approx \frac{1}{\lambda_{2}}\left(\ln \left(e_{2}(0)\right)-\ln \delta\right) .
$$

Summarizing the above contents, the total convergence time for velocity channel is

$$
t_{1}=t_{11}+t_{12}+t_{13}
$$

Likewise, the total approximate convergence time for altitude channel (in fact, the altitude channel is asymptotic convergence) is

$$
t_{2}=t_{21}+t_{22}+t_{23}+t_{e_{2}}
$$

In other words, it is obtained that

$$
\begin{aligned}
& e_{1}=V-V_{d}=0, \quad \forall t>t_{1}, \\
& e_{2}=h-h_{d}=0, \quad \forall t>t_{2} \longrightarrow \infty .
\end{aligned}
$$

In a word, the system output variables $V$ and $h$ can track the velocity command $V_{d}$ and altitude command $h_{d}$ and afterwards keep stable under the proposed controller in (19), respectively. Furthermore, in accordance with the character of the sliding mode control, the controller is robust in the presence of parameter uncertainties. The proof is completed.

Remark 9. It is worth noting the terms $\Psi_{1}$ and $\Psi_{2}$ in the location of the denominator for controller (19). They may cause singularity problem once the convergence speed of sliding mode surface equals zero. Hence, the following constraint is given:

$$
\Psi_{i}= \begin{cases}\Psi_{i}, & \left|\Psi_{i}\right|>\varepsilon \\ \varepsilon, & \left|\Psi_{i}\right| \leq \varepsilon\end{cases}
$$

where $\varepsilon$ is a small positive constant, $i=1,2$.

Remark 10. There are three layers of sliding mode manifolds for each channel. In each channel, these sliding surfaces are recursive. The last layer sliding surface first arrives; in this moment, the system states start to move toward the secondary sliding surface. After a period of time, the second layer sliding surface arrives. Then, each sliding surface arrives successively. Finally, the system tracking error converges to zero in limited time. The flowchart of these three layers of sliding mode manifolds is shown in Figure 1.

Remark 11. Taking into account (22)-(23) and (27)-(28), the fractional powers are $2-1 / a_{i}$ and $1-1 / a_{i}$. Because $1<2-$ $1 / a_{i}<2,0<1-1 / a_{i}<1$ for $a_{i}>1, i=1,2$, there is no negative fractional power in every procedure. Therefore, the singularity problem existing in terminal sliding mode (TSM) is avoided in the proposed controller. 


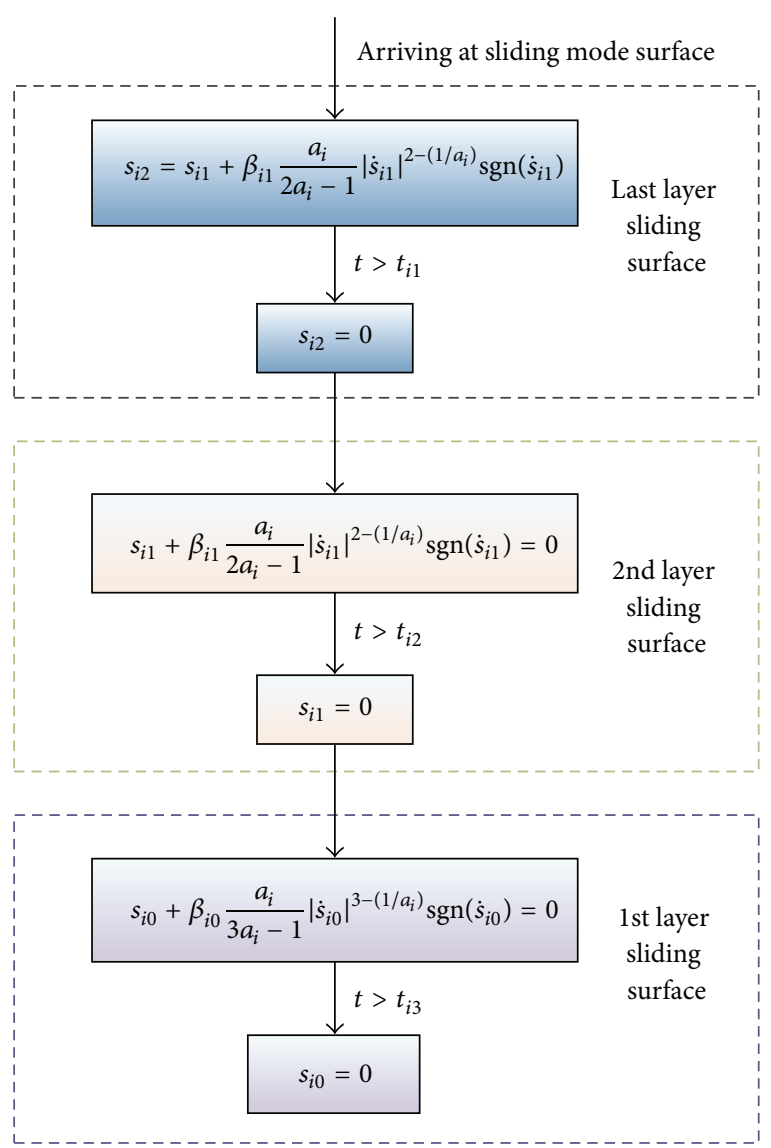

FIgURE 1: The flowchart of recursive sliding manifolds.

Remark 12. Although there is sign function in controller (19), the controller is continuous; namely, the chattering problem that usually existed in traditional sliding mode control is eliminated. This is due to the term $k_{i 2}\left|s_{i 2}\right|^{\rho_{i}} \operatorname{sgn}\left(s_{i 2}\right), i=1,2$, which is time continuous and removing the discontinuity.

3.2. Antiwindup Controller. For the actuator saturation problem of HFV, an antiwindup controller is presented in this subsection.

When the actuator saturation is taken into account and the external disturbance is not, (12) can be rewritten as

$$
\left[\begin{array}{c}
\dddot{V} \\
h^{(4)}
\end{array}\right]=\left[\begin{array}{c}
F_{V} \\
F_{h}
\end{array}\right]+\mathbf{B} \cdot \operatorname{sat}(\mathbf{u}),
$$

where $\operatorname{sat}(\mathbf{u})=\left[\begin{array}{l}\operatorname{sat}\left(\beta_{c}\right) \\ \operatorname{sat}\left(\delta_{e}\right)\end{array}\right]$.

The saturation functions of actuators are defined as

$$
\begin{aligned}
& \operatorname{sat}\left(\beta_{c}\right)= \begin{cases}\operatorname{sgn}\left(\beta_{c}\right), & \left|\beta_{c}\right|>\beta^{*} \\
\beta_{c}, & \left|\beta_{c}\right| \leq \beta^{*},\end{cases} \\
& \operatorname{sat}\left(\delta_{e}\right)= \begin{cases}\operatorname{sgn}\left(\delta_{e}\right), & \left|\delta_{e}\right|>\delta_{e}^{*} \\
\delta_{e}, & \left|\delta_{e}\right| \leq \delta_{e}^{*},\end{cases}
\end{aligned}
$$

where $\beta^{*}$ and $\delta_{e}^{*}$ are saturation value of throttle setting and elevator deflection, respectively.

Define the following saturation nonlinear functions as

$$
\begin{aligned}
& S_{a}\left(\beta_{c}\right)=\beta_{c}-\operatorname{sat}\left(\beta_{c}\right), \\
& S_{a}\left(\delta_{e}\right)=\delta_{e}-\operatorname{sat}\left(\delta_{e}\right) .
\end{aligned}
$$

Then, the actuator saturation functions are described as

$$
\left[\begin{array}{l}
\operatorname{sat}\left(\beta_{c}\right) \\
\operatorname{sat}\left(\delta_{e}\right)
\end{array}\right]=\mathbf{u}-\left[\begin{array}{l}
S_{a}\left(\beta_{c}\right) \\
S_{a}\left(\delta_{e}\right)
\end{array}\right]
$$

Therefore, (47) is changed for

$$
\left[\begin{array}{c}
\dddot{V} \\
h^{(4)}
\end{array}\right]=\left[\begin{array}{c}
F_{V} \\
F_{h}
\end{array}\right]+\mathbf{B u}-\mathbf{B}\left[\begin{array}{c}
S_{a}\left(\beta_{c}\right) \\
S_{a}\left(\delta_{e}\right)
\end{array}\right] .
$$

Theorem 13. Consider the linearized model (51) under the control constraints and without external disturbance; the system can stably converge to the reference commands $V_{d}$ and $h_{d}$, if the controller is chosen as

$\mathbf{u}$

$$
\begin{aligned}
= & \mathbf{B}^{-1}\left[\begin{array}{l}
\frac{\left(-k_{11} s_{12}-k_{12}\left|s_{12}\right|^{\rho_{1}} \operatorname{sgn}\left(s_{12}\right)-\Phi_{1}\right)}{\Psi_{1}}+\dddot{V}_{d}-F_{V} \\
\frac{\left(-k_{21} s_{22}-k_{22}\left|s_{22}\right|^{\rho_{2}} \operatorname{sgn}\left(s_{22}\right)-\Phi_{2}\right)}{\Psi_{2}}+h_{d}^{(4)}-F_{h}
\end{array}\right] \\
& +\mathbf{B}^{-1}\left[\begin{array}{l}
\kappa_{11}(b) S_{a}\left(\beta_{c}\right)+\kappa_{12}(b) S_{a}\left(\delta_{e}\right) \\
\kappa_{21}(b) S_{a}\left(\beta_{c}\right)+\kappa_{22}(b) S_{a}\left(\delta_{e}\right)
\end{array}\right],
\end{aligned}
$$

where

$$
\begin{gathered}
\kappa_{i 1}(b)= \begin{cases}0, & S_{a}\left(\beta_{c}\right)=0 \\
b_{i 1}, & S_{a}\left(\beta_{c}\right) \neq 0,\end{cases} \\
\kappa_{i 2}(b)= \begin{cases}0, & S_{a}\left(\delta_{e}\right)=0 \\
b_{i 2}, & S_{a}\left(\delta_{e}\right) \neq 0,\end{cases}
\end{gathered}
$$

Proof. Substituting (51) into (31) yields

$$
\begin{aligned}
{\left[\begin{array}{c}
\dot{s}_{12} \\
\dot{s}_{22}
\end{array}\right] } & =\left[\begin{array}{l}
\Phi_{1} \\
\Phi_{2}
\end{array}\right]+\left[\begin{array}{cc}
\Psi_{1} & 0 \\
0 & \Psi_{2}
\end{array}\right]\left(\left[\begin{array}{c}
F_{V} \\
F_{h}
\end{array}\right]+\mathbf{B u}\right. \\
-\mathbf{B} & {\left.\left[\begin{array}{l}
S_{a}\left(\beta_{c}\right) \\
S_{a}\left(\delta_{e}\right)
\end{array}\right]-\left[\begin{array}{c}
\dddot{V}_{d} \\
h_{d}^{(4)}
\end{array}\right]\right) . }
\end{aligned}
$$


Then, substituting the proposed controller (52) into (54), we acquire

$$
\begin{aligned}
& {\left[\begin{array}{c}
\dot{s}_{12} \\
\dot{s}_{22}
\end{array}\right]=\left[\begin{array}{l}
-k_{11} s_{12}-k_{12}\left|s_{12}\right|^{\rho_{1}} \operatorname{sgn}\left(s_{12}\right) \\
-k_{21} s_{22}-k_{22}\left|s_{22}\right|^{\rho_{2}} \operatorname{sgn}\left(s_{22}\right)
\end{array}\right]} \\
& +\left[\begin{array}{cc}
\Psi_{1} & 0 \\
0 & \Psi_{2}
\end{array}\right]\left(\left[\begin{array}{l}
\kappa_{11}(b) S_{a}\left(\beta_{c}\right)+\kappa_{12}(b) S_{a}\left(\delta_{e}\right) \\
\kappa_{21}(b) S_{a}\left(\beta_{c}\right)+\kappa_{22}(b) S_{a}\left(\delta_{e}\right)
\end{array}\right]\right. \\
& \left.-\mathbf{B}\left[\begin{array}{l}
S_{a}\left(\beta_{c}\right) \\
S_{a}\left(\delta_{e}\right)
\end{array}\right]\right) .
\end{aligned}
$$

In terms of the definition $\kappa_{i j}(b)$, we can obtain

$$
\begin{aligned}
& {\left[\begin{array}{l}
\kappa_{11}(b) S_{a}\left(\beta_{c}\right)+\kappa_{12}(b) S_{a}\left(\delta_{e}\right) \\
\kappa_{21}(b) S_{a}\left(\beta_{c}\right)+\kappa_{22}(b) S_{a}\left(\delta_{e}\right)
\end{array}\right]-\mathbf{B}\left[\begin{array}{l}
S_{a}\left(\beta_{c}\right) \\
S_{a}\left(\delta_{e}\right)
\end{array}\right]} \\
& \quad=\left[\begin{array}{l}
\left(\kappa_{11}(b)-b_{11}\right) S_{a}\left(\beta_{c}\right)+\left(\kappa_{12}(b)-b_{12}\right) S_{a}\left(\delta_{e}\right) \\
\left(\kappa_{21}(b)-b_{21}\right) S_{a}\left(\beta_{c}\right)+\left(\kappa_{22}(b)-b_{22}\right) S_{a}\left(\delta_{e}\right)
\end{array}\right] \\
& =0 .
\end{aligned}
$$

So, (55) is changed for

$$
\left[\begin{array}{l}
\dot{s}_{12} \\
\dot{s}_{22}
\end{array}\right]=\left[\begin{array}{l}
-k_{11} s_{12}-k_{12}\left|s_{12}\right|^{\rho_{1}} \operatorname{sgn}\left(s_{12}\right) \\
-k_{21} s_{22}-k_{22}\left|s_{22}\right|^{\rho_{2}} \operatorname{sgn}\left(s_{22}\right)
\end{array}\right] \text {. }
$$

In accordance with the proof process of Theorem 8, the system state will arrive at each layer of the sliding mode surface in sequence, and finally the states will be stable at equilibrium point. Then, the system can stably converge to its reference command under control saturation. This completes the proof.

Remark 14. The antiwindup controller in (52) consists of the CRSMC tracking controller in (19) and the compensation factor, which is the second part on the right of (52). The compensation factor can compensate the lost part of the control variable due to the actuator saturation.

3.3. Improved Extended Disturbance Observer. According to the external disturbance of the system, a disturbance observer based on EDO is presented.

Defining $x_{1}=\ddot{V}, x_{2}=h^{(3)}$, then (10) is converted to

$$
\begin{aligned}
& \dot{x}_{1}=F_{V}+B_{1 u}+D_{1}, \\
& \dot{x}_{2}=F_{h}+B_{2 u}+D_{2},
\end{aligned}
$$

where

$$
\begin{aligned}
B_{1 u} & =b_{11} \beta_{c}+b_{12} \delta_{e}, \\
B_{2 u} & =b_{21} \beta_{c}+b_{22} \delta_{e}, \\
b_{11} d_{1}(t)+b_{12} d_{2}(t) & =D_{1}, \\
b_{21} d_{1}(t)+b_{22} d_{2}(t) & =D_{2} .
\end{aligned}
$$

According to the pseudo system state equation of (58) which has disturbance in all channels, the EDO is designed as

$$
\begin{aligned}
\widehat{D}_{i}^{(j-1)} & =p_{i j}+l_{i j} x_{i} \\
\dot{p}_{i j} & =-l_{i j}\left(F_{m}+B_{i u}+\widehat{D}_{i}\right)+\zeta_{j} \widehat{D}_{i}^{(j)}
\end{aligned}
$$

where

$$
\zeta_{j}=\left\{\begin{array}{ll}
1, & j \neq r \\
0, & j=r
\end{array},\right.
$$

$$
i=1,2, m=V, h, j=1,2, \ldots, r \text {, }
$$

$r$ is the order of EDO, $p_{i j}$ are the auxiliary variables, and $l_{i j}$ are the gain parameters chosen by designer.

In [28], every gain parameter should be regulated independently, which is inconvenient when the number of parameters is giant. The improved EDO (IEDO) presented in this paper simplifies the regulating process; afterwards, there is only one parameter that needs to be regulated, which is inspired by Gao in $[27,33]$.

For $r(r \geq 3)$ order EDO, the EDO of (61) is expressed as follows:

$$
\begin{gathered}
\widehat{D}_{i}=p_{i 1}+l_{i 1} x_{i}, \\
\dot{p}_{i 1}=-l_{i 1}\left(F_{m}+B_{i u}+\widehat{D}_{i}\right)+\widehat{\dot{D}}_{i}, \\
\widehat{\dot{D}}_{i}=p_{i 2}+l_{i 2} x_{i}, \\
\dot{p}_{i 2}=-l_{i 2}\left(F_{m}+B_{i u}+\widehat{D}_{i}\right)+\widehat{\vec{D}}_{i}, \\
\vdots \\
\widehat{D}_{i}^{(r-1)}=p_{i r}+l_{i r} x_{i}, \\
=-l_{i r}\left(F_{m}+B_{i u}+\widehat{D}_{i}\right) .
\end{gathered}
$$

Define the estimation error of disturbance and its derivative as

$$
\begin{aligned}
& \widetilde{D}_{i}=D_{i}-\widehat{D}_{i}, \\
& \widetilde{D}_{i}=\dot{D}_{i}-\widehat{\dot{D}}_{i}, \\
& \widetilde{D}_{i}=\ddot{D}_{i}-\widehat{\ddot{D}}_{i},
\end{aligned}
$$

$$
\widetilde{D}_{i}^{(r-1)}=D_{i}^{(r-1)}-\widehat{D}_{i}^{(r-1)}
$$

Taking the first time derivative of (63) and substituting (64) into it yield

$$
\dot{\widehat{D}}_{i}=l_{i 1} \widetilde{D}_{i}+\widehat{\dot{D}}_{i}
$$


Subtracting both sides of (70) from $\dot{D}_{i}$, we acquire

$$
\dot{\widetilde{D}}_{i}=\dot{D}_{i}-\widehat{\dot{D}}_{i}=-l_{i 1} \widetilde{D}_{i}+\widetilde{\dot{D}}_{i}
$$
(68):

Similarly, the following equations are obtained from (65)-

$$
\begin{gathered}
\dot{\vec{D}}_{i}=-l_{i 2} \widetilde{D}_{i}+\widetilde{\vec{D}}_{i}, \\
\vdots \\
\dot{\widetilde{D}}_{i}^{(r-1)}=-l_{i r} \widetilde{D}_{i}+D_{i}^{(r)} .
\end{gathered}
$$

Then, (71)-(73) are written together as

$$
\dot{\widetilde{\mathbf{E}}}_{i}=\mathbf{A} \widetilde{\mathbf{E}}_{i}+\mathbf{C} D_{i}^{(r)},
$$

where

$$
\begin{aligned}
\widetilde{\mathbf{E}}_{i} & =\left[\begin{array}{c}
\dot{\widetilde{D}}_{i} \\
\dot{\vec{D}}_{i} \\
\vdots \\
\dot{\vec{D}}_{i}^{(r-1)}
\end{array}\right], \\
\mathbf{A} & =\left[\begin{array}{ccccc}
-l_{i 1} & 1 & \cdots & \cdots & 0 \\
-l_{i 2} & 0 & 1 & \cdots & 0 \\
\vdots & \ldots & \ldots & \cdots & 1 \\
-l_{i r} & 0 & \cdots & \cdots & 0
\end{array}\right], \\
\mathbf{C} & =\left[\begin{array}{c}
0 \\
0 \\
\vdots \\
1
\end{array}\right] .
\end{aligned}
$$

Then, the gain parameters are calculated as follows:

$$
|\lambda \mathbf{I}-\mathbf{A}|=\left(\lambda+\omega_{0 i}\right)^{r},
$$

where $\mathbf{I}$ is unit matrix and $\omega_{0 i}$ is the observer bandwidth.

Therefore, every gain parameter is only determined by $\omega_{0 i}$, which simplifies the regulation process. For a secondorder EDO as an example, the gain parameters are reduced

$$
\begin{aligned}
|\lambda \mathbf{I}-\mathbf{A}| & =\left|\begin{array}{cc}
\lambda+l_{i 1} & -1 \\
l_{i 2} & \lambda
\end{array}\right|=\lambda^{2}+l_{i 1} \lambda+l_{i 2}=\left(\lambda+\omega_{0 i}\right)^{2} \\
& =\lambda^{2}+2 \omega_{0 i} \lambda+\omega_{0 i}^{2} .
\end{aligned}
$$

So the parameters are chosen to $l_{i 1}=2 \omega_{0 i}, l_{i 2}=\omega_{0 i}^{2}$.

In terms of [28] and Assumption 1, the norm of the estimation error $\widetilde{\mathbf{E}}_{i}$ is bounded after some certain time, which also means that the external disturbances $D_{1}$ and $D_{2}$ are estimated well after a certain time, namely, $\widehat{D}_{1}$ and $\widehat{D}_{2}$.

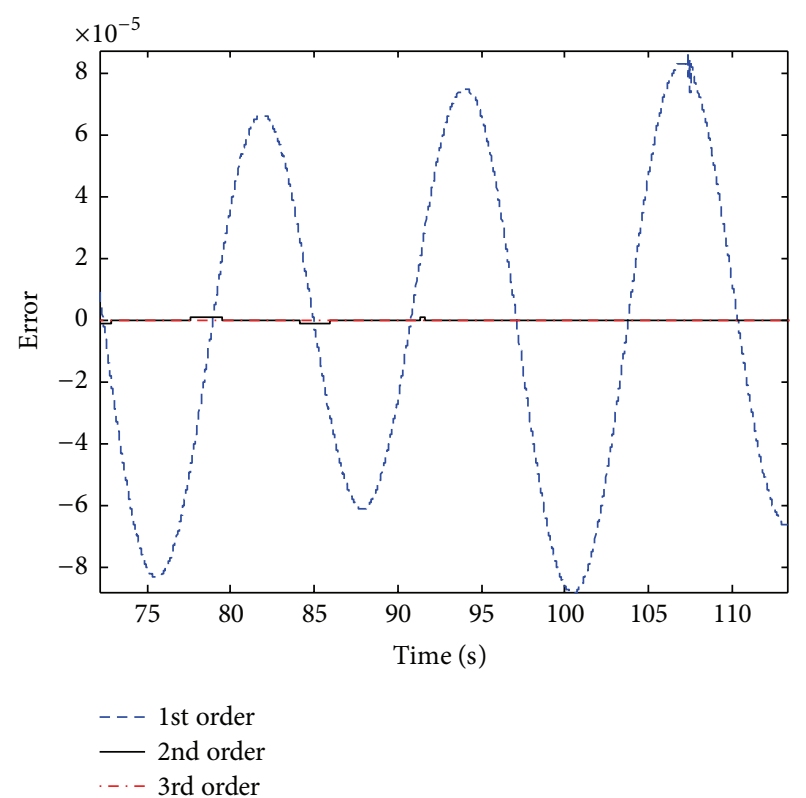

FIGURE 2: Comparison of estimation error.

By virtue of (60), the disturbances acting on the actuators are estimated as

$$
\left[\begin{array}{l}
\widehat{d}_{1}(t) \\
\widehat{d}_{2}(t)
\end{array}\right]=\mathbf{B}^{-1}\left[\begin{array}{l}
\widehat{D}_{1} \\
\widehat{D}_{2}
\end{array}\right] .
$$

Remark 15. For IEDO, different estimation precision is obtained from different orders, and different orders of IEDO have different estimation time. According to three kinds of IEDO, simulations are conducted for comparing. The simulation results are shown in Figures 2 and 3.

Figure 2 reveals the estimation error, from which it is seen that the higher the order, the smaller the error. In Figure 3, it is shown that if the order is higher, the estimation time is longer. In other words, the higher estimation accuracy needs more time as a cost. Synthesizing Figures 2 and 3, the IEDO for HFV in this paper is chosen as second order, which is the compromise between the estimation accuracy and estimation time.

\section{Composite Controller and Stability Analysis}

In this section, the composite controller including CRSMC under control constraints and IEDO is analyzed. The external disturbance of system is estimated by IEDO, and the estimated value is used as compensator in CRSMC. Hence, the composite controller is designed as

$\mathbf{u}$

$$
=\mathbf{B}^{-1}\left[\begin{array}{l}
\frac{\left(-k_{11} s_{12}-k_{12}\left|s_{12}\right|^{\rho_{1}} \operatorname{sgn}\left(s_{12}\right)-\Phi_{1}\right)}{\Psi_{1}}+\dddot{V}_{d}-F_{V} \\
\frac{\left(-k_{21} s_{22}-k_{22}\left|s_{22}\right|^{\rho_{2}} \operatorname{sgn}\left(s_{22}\right)-\Phi_{2}\right)}{\Psi_{2}}+h_{d}^{(4)}-F_{h}
\end{array}\right]
$$



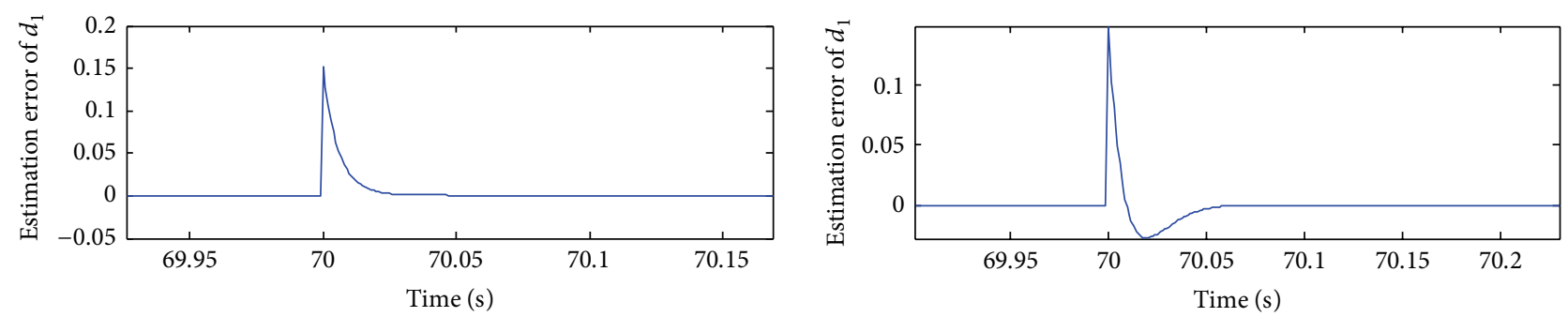

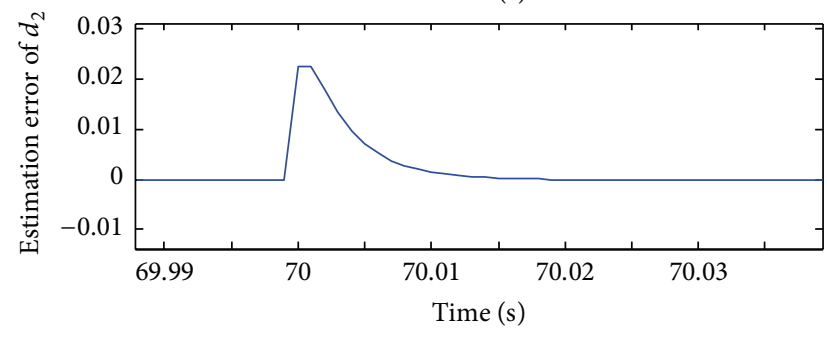

(a) First order

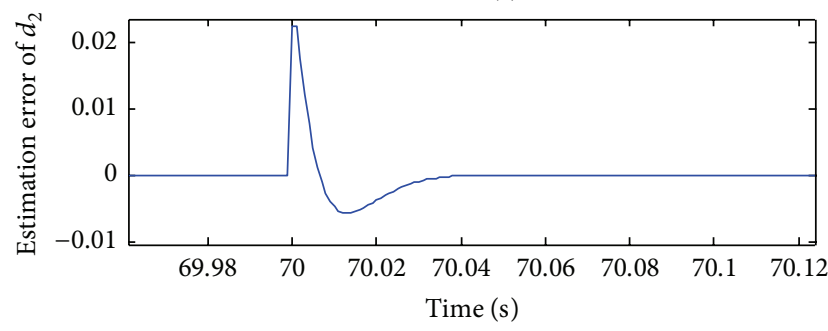

(b) Second order
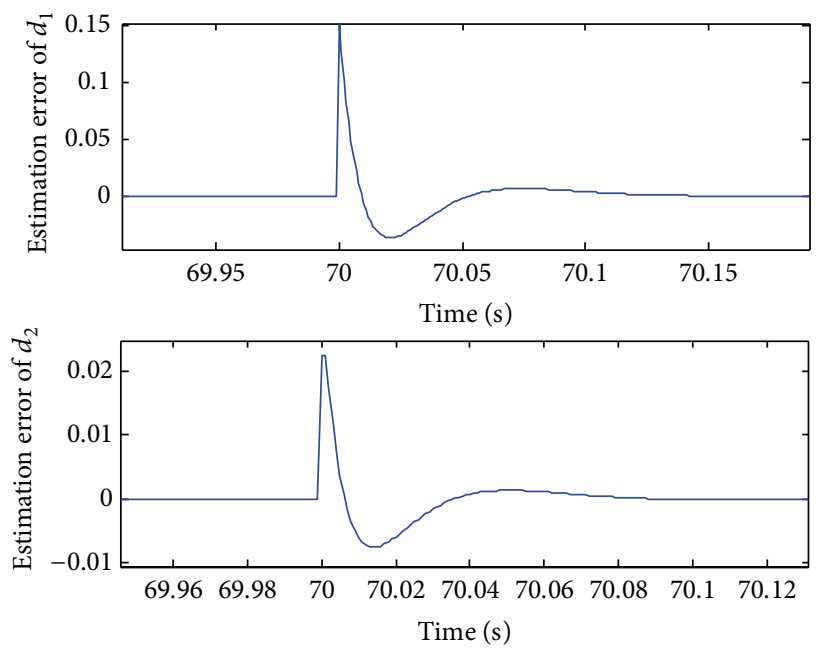

(c) Third order

FIGURE 3: Comparison of estimation time.

$$
+\mathbf{B}^{-1}\left[\begin{array}{l}
\kappa_{11}(b) S_{a}\left(\beta_{c}\right)+\kappa_{12}(b) S_{a}\left(\delta_{e}\right) \\
\kappa_{21}(b) S_{a}\left(\beta_{c}\right)+\kappa_{22}(b) S_{a}\left(\delta_{e}\right)
\end{array}\right]-\left[\begin{array}{l}
\widehat{d}_{1}(t) \\
\widehat{d}_{2}(t)
\end{array}\right]
$$

Theorem 16. Taking into account the longitudinal dynamics of HFV model (1) in the presence of multiple uncertainties under control constraints, the system is stable and robust if the controller is chosen as (79).

Proof. Choose the Lyapunov candidate function

$$
L_{a}=\frac{1}{2} s^{T} s
$$

Considering multiple uncertainties and actuator saturation, it is obtained from (12) that

$$
\left[\begin{array}{c}
\dddot{V} \\
h^{(4)}
\end{array}\right]=\left[\begin{array}{c}
F_{V} \\
F_{h}
\end{array}\right]+\mathbf{B} \cdot \operatorname{sat}(\mathbf{u})+\mathbf{B}\left[\begin{array}{l}
d_{1}(t) \\
d_{2}(t)
\end{array}\right] .
$$

Substituting (81) into (31), we obtain

$$
\begin{aligned}
& {\left[\begin{array}{c}
\dot{s}_{12} \\
\dot{s}_{22}
\end{array}\right]=\left[\begin{array}{l}
\Phi_{1} \\
\Phi_{2}
\end{array}\right]+\left[\begin{array}{cc}
\Psi_{1} & 0 \\
0 & \Psi_{2}
\end{array}\right]\left(\left[\begin{array}{c}
F_{V} \\
F_{h}
\end{array}\right]-\left[\begin{array}{c}
\dddot{V}_{d} \\
h_{d}^{(4)}
\end{array}\right]+\mathbf{B u}\right.} \\
& \left.-\mathbf{B}\left[\begin{array}{l}
S_{a}\left(\beta_{c}\right) \\
S_{a}\left(\delta_{e}\right)
\end{array}\right]+\mathbf{B}\left[\begin{array}{l}
d_{1}(t) \\
d_{2}(t)
\end{array}\right]\right) .
\end{aligned}
$$

Then, taking first-order derivative of (80) along the dynamics of (82) yields

$$
\begin{aligned}
\dot{L}_{a} & =s^{T} \dot{s}=s^{T}\left[\left[\begin{array}{l}
\Phi_{1} \\
\Phi_{2}
\end{array}\right]+\left[\begin{array}{cc}
\Psi_{1} & 0 \\
0 & \Psi_{2}
\end{array}\right]\left(\left[\begin{array}{l}
F_{V} \\
F_{h}
\end{array}\right]-\left[\begin{array}{c}
\dddot{V}_{d} \\
h_{d}^{(4)}
\end{array}\right]\right.\right. \\
& \left.\left.+\mathbf{B u}-\mathbf{B}\left[\begin{array}{l}
S_{a}\left(\beta_{c}\right) \\
S_{a}\left(\delta_{e}\right)
\end{array}\right]+\mathbf{B}\left[\begin{array}{l}
d_{1}(t) \\
d_{2}(t)
\end{array}\right]\right)\right] .
\end{aligned}
$$




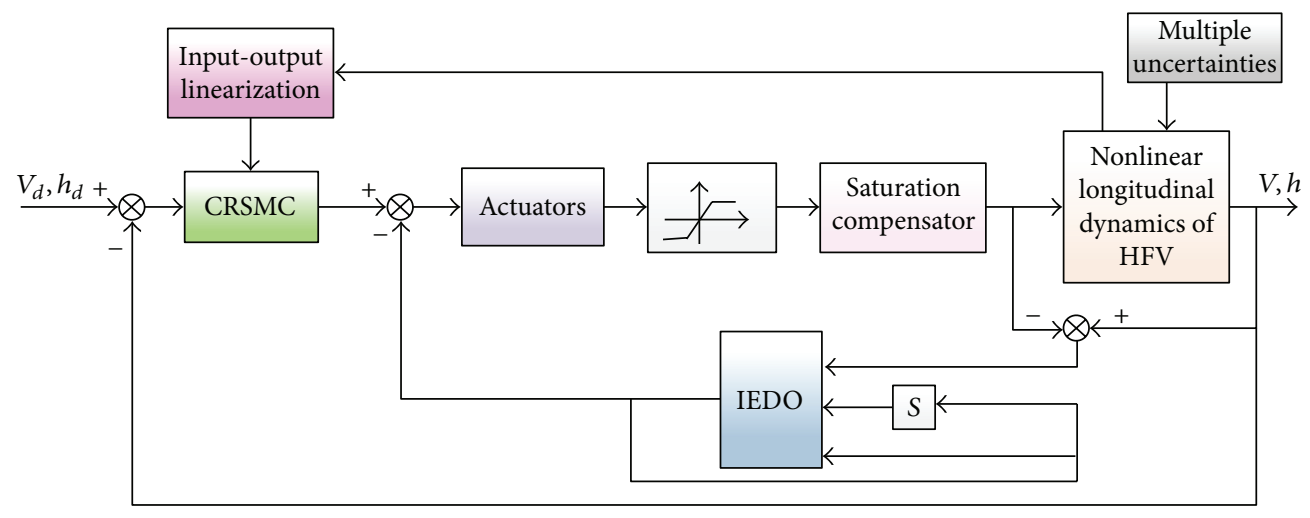

FIgURE 4: Structure diagram of composite control scheme.

Substituting controller (79) into (83), we acquire

$$
\begin{aligned}
\dot{L}_{a} & =s^{T}\left(\left[\begin{array}{l}
-k_{11} s_{12}-k_{12}\left|s_{12}\right|^{\rho_{1}} \operatorname{sgn}\left(s_{12}\right) \\
-k_{21} s_{22}-k_{22}\left|s_{22}\right|^{\rho_{2}} \operatorname{sgn}\left(s_{22}\right)
\end{array}\right]\right. \\
& \left.+\left[\begin{array}{cc}
\Psi_{1} & 0 \\
0 & \Psi_{2}
\end{array}\right]\left(\Delta \mathbf{B}\left[\begin{array}{l}
S_{a}\left(\beta_{c}\right) \\
S_{a}\left(\delta_{e}\right)
\end{array}\right]+\mathbf{B}\left[\begin{array}{c}
\tilde{d}_{1}(t) \\
\tilde{d}_{2}(t)
\end{array}\right]\right)\right),
\end{aligned}
$$

where

$$
\begin{aligned}
\Delta \mathbf{B} & =\left[\begin{array}{ll}
\kappa_{11}(b)-b_{11} & \kappa_{12}(b)-b_{12} \\
\kappa_{21}(b)-b_{21} & \kappa_{22}(b)-b_{22}
\end{array}\right], \\
\widetilde{d}_{i}(t) & =d_{i}(t)-\widehat{d}_{i}(t), \quad i=1,2,
\end{aligned}
$$

is the disturbance evaluated error.

In accordance with IEDO, the evaluated error can be arbitrarily small after some extent time, that is, $t_{a}$, when the error is approximately regarded as zero. In addition, it is known that $\Delta \mathbf{B}\left[S_{a}\left(\beta_{c}\right) S_{a}\left(\delta_{e}\right)\right]^{T}=0$ by means of Theorem 13. Therefore, $\forall t>t_{a}$, (84) is changed as

$$
\begin{aligned}
\dot{L}_{a} & =s^{T}\left[\begin{array}{l}
-k_{11} s_{12}-k_{12}\left|s_{12}\right|^{\rho_{1}} \operatorname{sgn}\left(s_{12}\right) \\
-k_{21} s_{22}-k_{22}\left|s_{22}\right|^{\rho_{2}} \operatorname{sgn}\left(s_{22}\right)
\end{array}\right] \\
& =-k_{11} s_{12}^{2}-k_{12}\left|s_{12}\right|^{\rho_{1}+1}-k_{21} s_{22}^{2}-k_{22}\left|s_{22}\right|^{\rho_{2}+1} \\
& \leq 0 .
\end{aligned}
$$

Hence, the sliding mode manifolds $s_{i 2}, i=1,2$, will be equal to zero in finite time. According to Theorem 8, the recursive sliding mode manifolds will arrive in turn. Finally, the tracking errors of velocity and altitude will converge to zero; at the same time, the system is stable and robust to disturbance. The proof is completed.

Remark 17. The estimated time of IEDO is governed by observer gain. So the time can be tuned enough small such that its effect on the system convergence time is little. Hence, the estimated time can be regulated to some extent in Theorem 16.
TABLE 1: Some parameters about simulation.

\begin{tabular}{lccc}
\hline Parameter & Notation & Value & Unit \\
\hline Mass & $m$ & 9375 & Slug \\
Moment of inertia & $I_{y y}$ & $7 \times 10^{6}$ & Slug. $\mathrm{ft}^{3}$ \\
Reference area & $S$ & 3603 & $\mathrm{ft}^{2}$ \\
Mean aerodynamic chord & $\bar{c}$ & 80 & $\mathrm{ft}$ \\
Elevator coefficient & $c_{e}$ & 0.0292 & Dimensionless \\
Density of air & $\rho$ & $2.4325 \times 10^{-5}$ & Slug $/ \mathrm{ft}^{3}$ \\
\hline
\end{tabular}

TABLE 2: Parameter uncertainties.

\begin{tabular}{lcccccc}
\hline Notation & $\Delta m$ & $\Delta I$ & $\Delta S$ & $\Delta \bar{c}$ & $\Delta \rho$ & $\Delta c_{e}$ \\
\hline Value & 0.03 & 0.02 & 0.03 & 0.02 & 0.03 & 0.02 \\
\hline
\end{tabular}

The structure diagram of composite control scheme for $\mathrm{HFV}$ in this paper is shown in Figure 4.

\section{Numerical Simulations}

In this section, the numerical simulations of the proposed scheme that consists of CRSMC and IEDO are conducted. The simulations are built in the trimmed cruise flight condition of HFV, in which the primary flight parameters are altitude $110000 \mathrm{ft}$, velocity $15060 \mathrm{ft} / \mathrm{s}$, Mach 15, angle of attack $0 \mathrm{rad}$, and pitch rate $0 \mathrm{rad} / \mathrm{s}$, respectively. The damping ratio and natural frequency of the engine dynamics are taken as 0.7 and $5 \mathrm{rad} / \mathrm{s}$, respectively. In the simulation, the reference command for velocity channel is step signal $100 \mathrm{ft} / \mathrm{s}$ and for altitude channel is $500 \mathrm{ft}$. Some other parameters about the simulation are listed in Table 1.

The parameter uncertainties in (5) are listed in Table 2.

The external disturbances in the simulation are chosen as

$$
\begin{aligned}
& d_{1}(t)=0.1 \sin (0.5 t)+0.2 \cos (0.3 t), \\
& d_{2}(t)=0.03 \sin (0.5 t)+0.01 \cos (0.3 t) .
\end{aligned}
$$

The above disturbances are added to the system at 70th second of simulation.

The simulation for HFV under the CRSMC tracking controller is first conducted. The simulation results are shown 


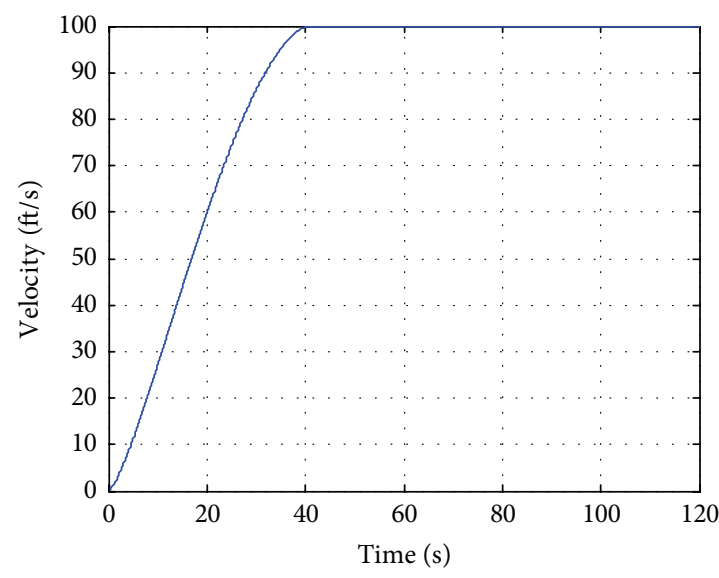

(a) Velocity

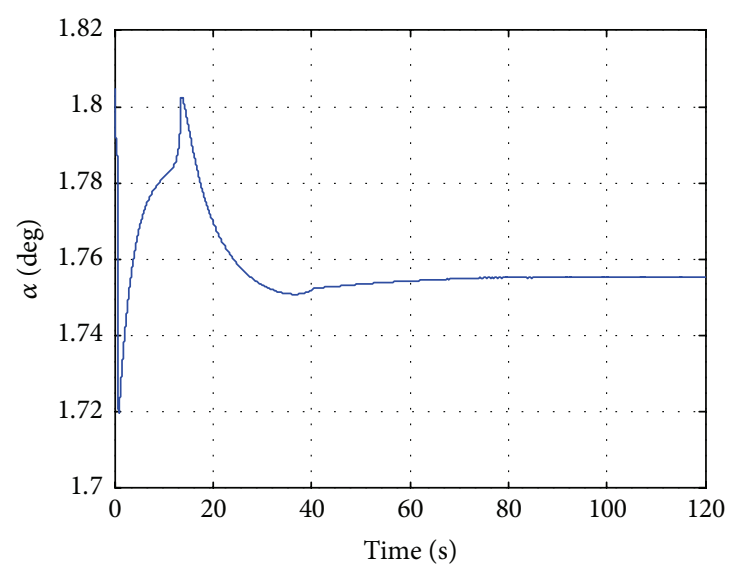

(c) Angle of attack

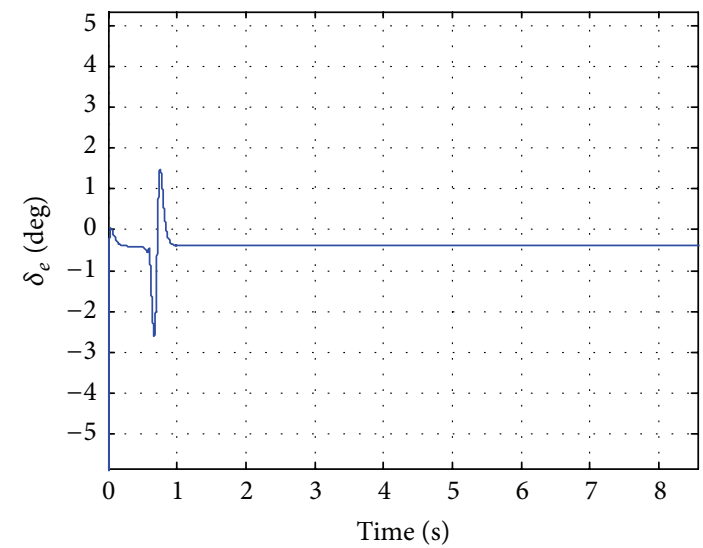

(e) Elevator deflection angle

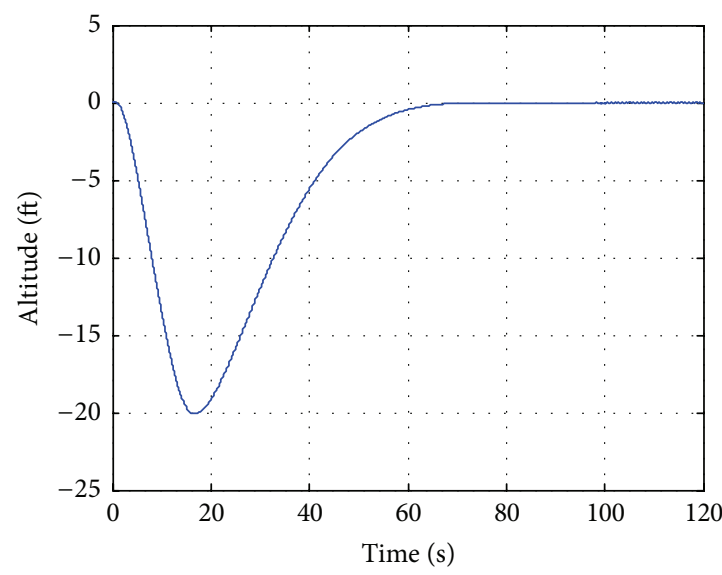

(b) Altitude

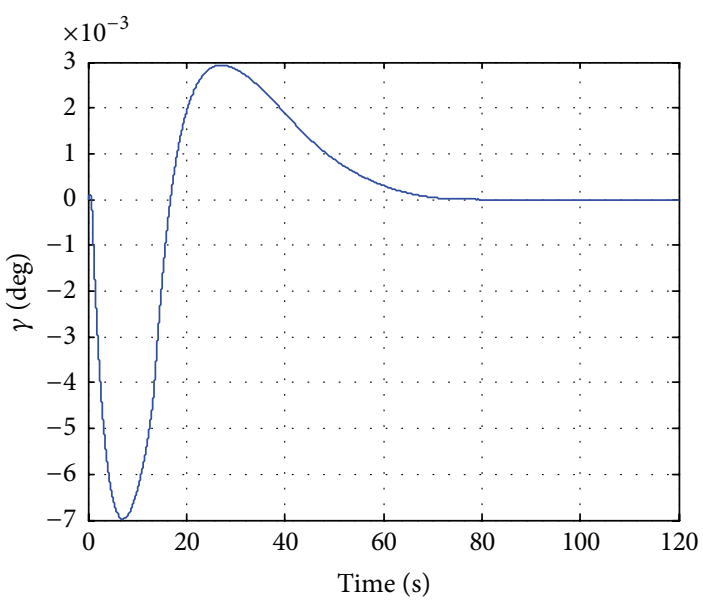

(d) Flight path angle

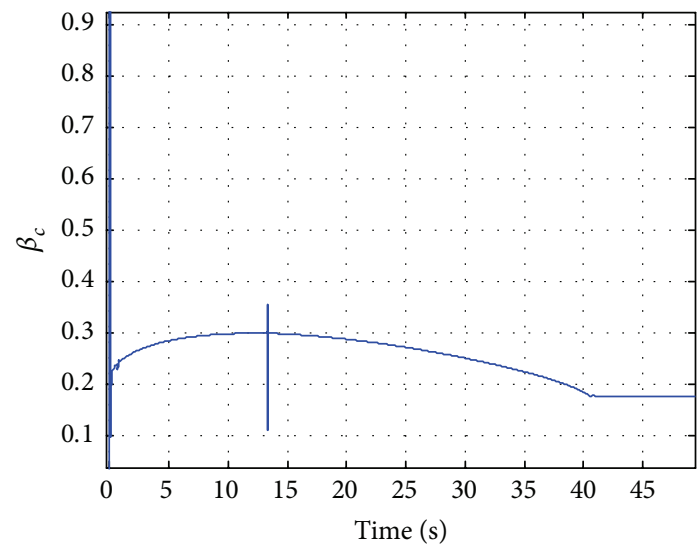

(f) Throttle setting

FIGURE 5: Response of velocity step command.

in Figures 5 and 6, in which Figure 5 displays the results of velocity channel and Figure 6 of altitude channel.

Figure 5(a) shows the velocity response for the velocity step command $100 \mathrm{ft} / \mathrm{s}$, from which it is seen that the velocity variable tracks the command at $40 \mathrm{~s}$ and then it keeps steady. The tracking speed of velocity variable remains constant, which demonstrates the finite time convergence of the proposed approach. Other parameter variables from Figures 5(b)-5(f) also achieve stability in finite time. Figures $5(\mathrm{e})$ and 5(f) are response curves of actuators, which is no chattering problem.

The altitude variable response for altitude step command $500 \mathrm{ft}$ is shown in Figure 6(b), from which the tracking time is about $50 \mathrm{~s}$. Then, the variable keeps steady. Other 


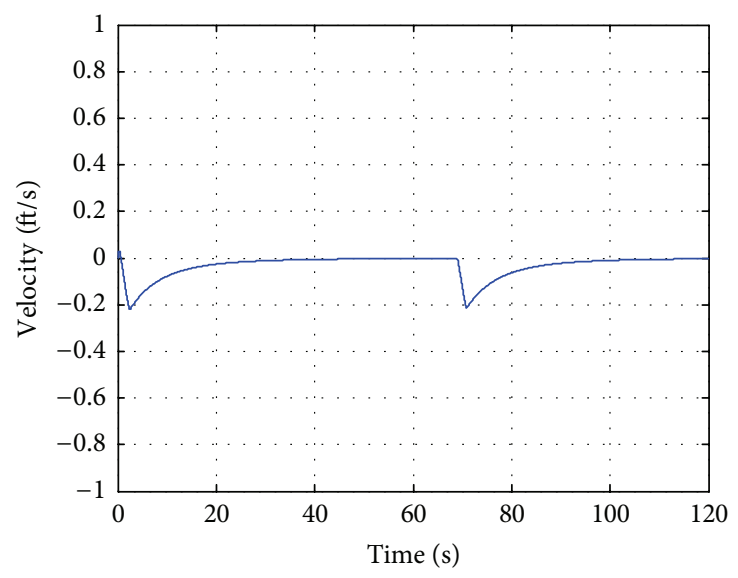

(a) Velocity

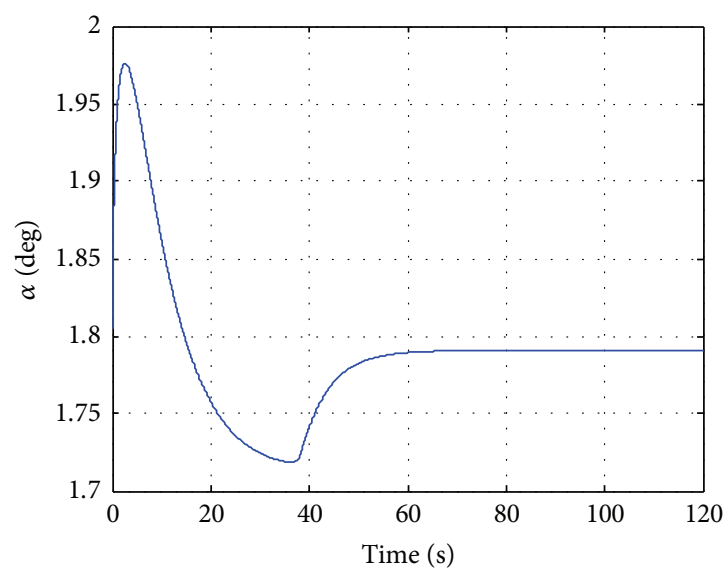

(c) Angle of attack

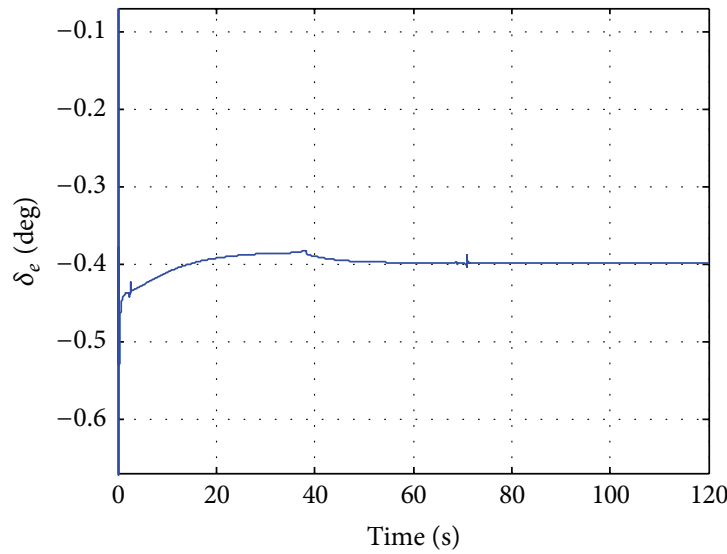

(e) Elevator deflection angle

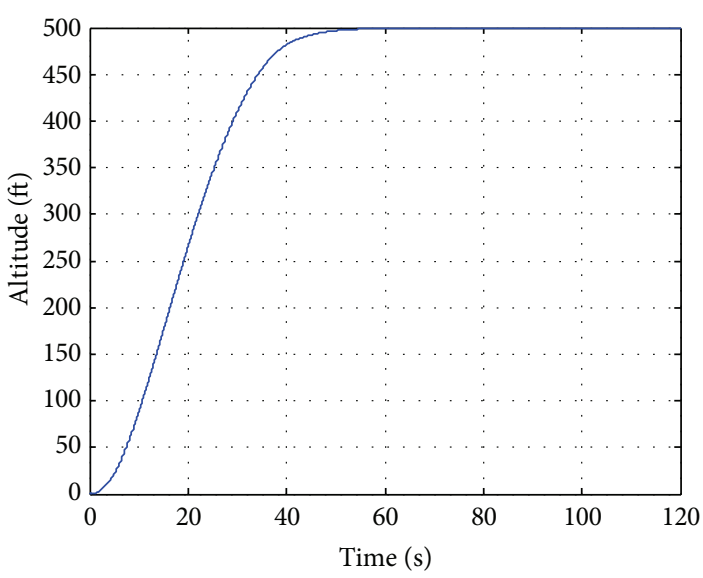

(b) Altitude

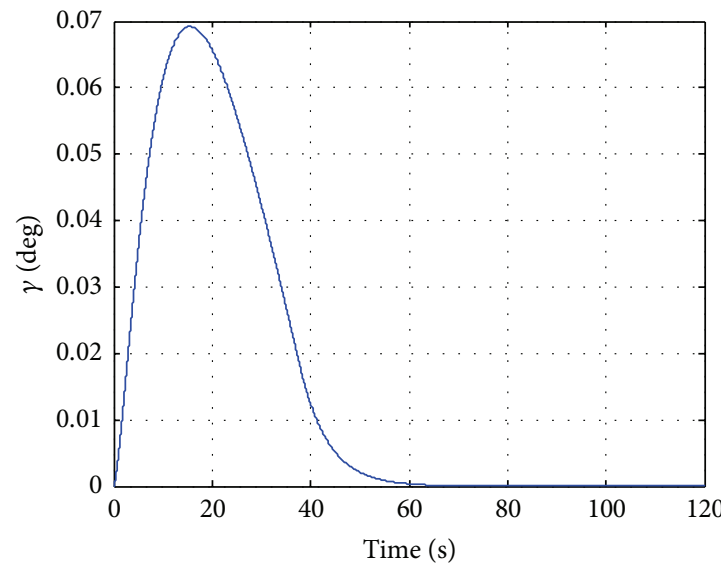

(d) Flight path angle

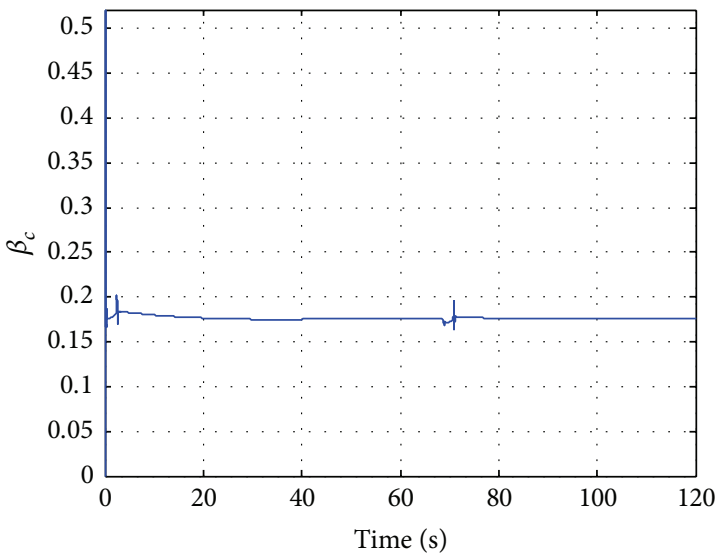

(f) Throttle setting

Figure 6: Response of altitude step command.

parameter curves from Figures 6(c)-6(f) indicate that the system achieves stable condition in finite time. It is worth noting that there is perturbation at about $70 \mathrm{~s}$. However, the perturbation is removed rapidly, which manifests the robustness of sliding mode control. The actuator response curves of Figures 6(e) and 6(f) depict no chattering problem.
In terms of Figures 5(e), 5(f), 6(e), and 6(f), it is demonstrated that the proposed scheme can eliminate chattering problem that usually existed in sliding mode control.

For comparing, the traditional sliding mode control with linear sliding surface is simulated for HFV, which consults [12]. The simulation results are shown in Figure 7. 

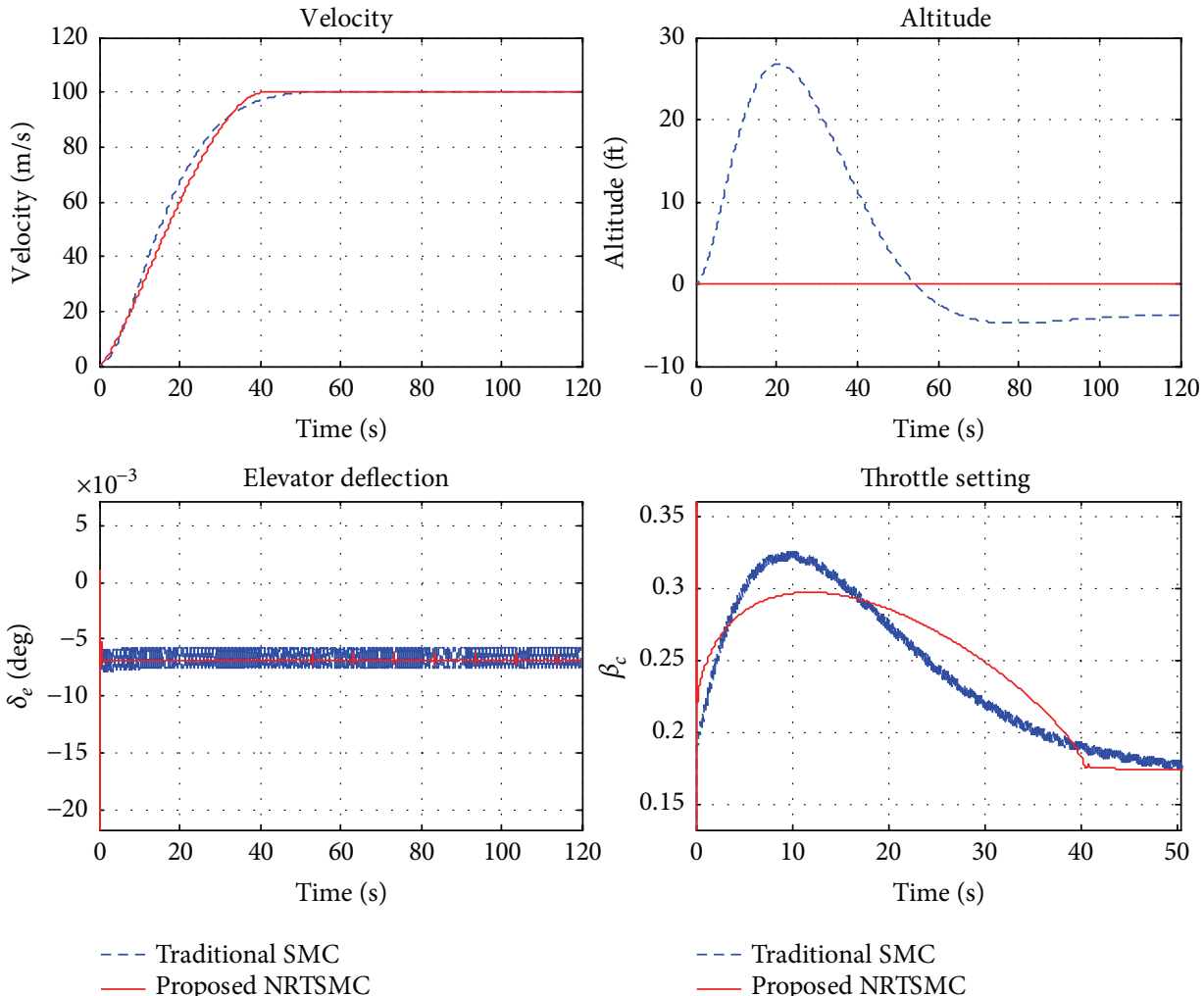

(a) Response for velocity step command
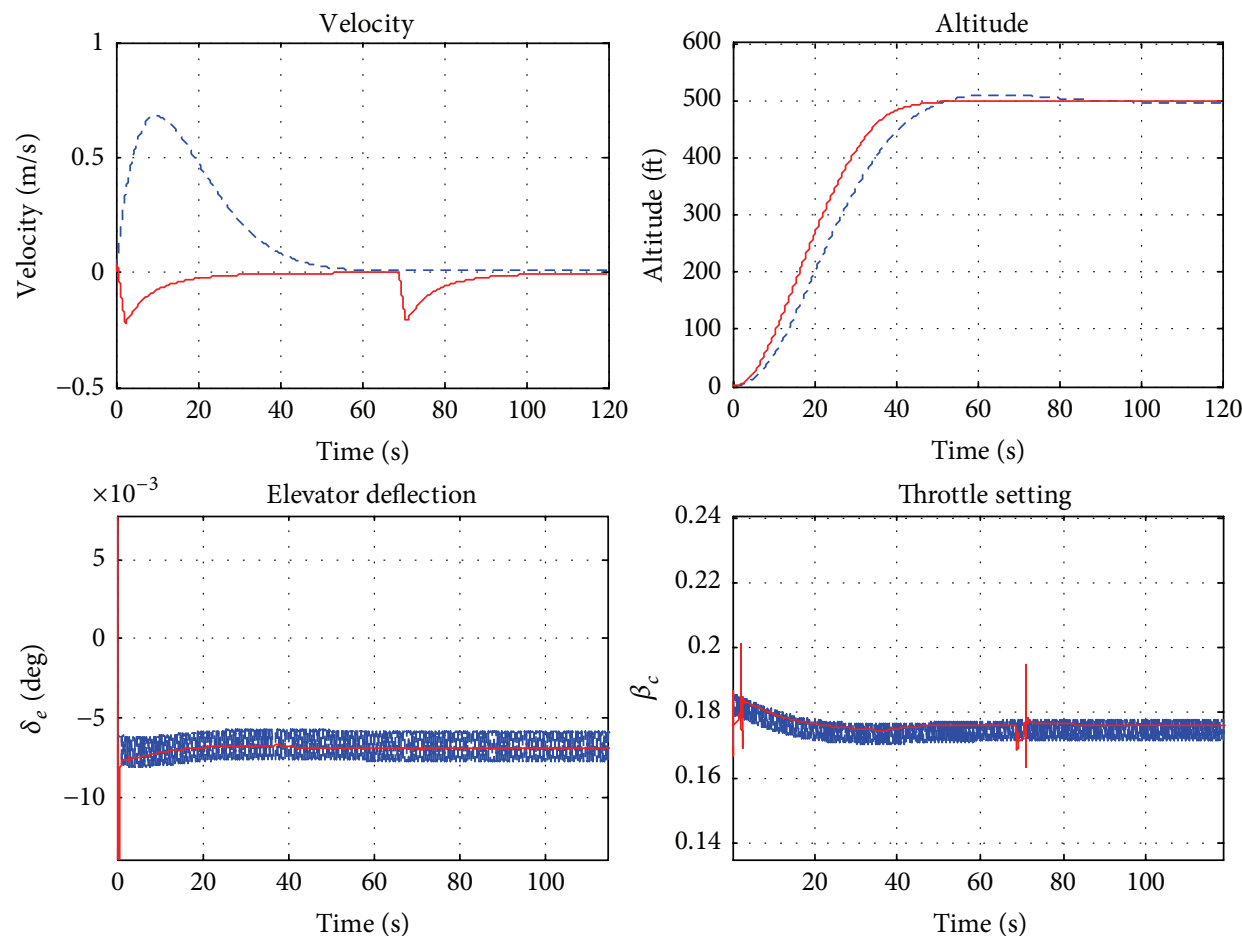

- - - Traditional SMC

- - Traditional SMC

— Proposed NRTSMC

- Proposed NRTSMC

(b) Response for altitude step command

FIGURE 7: Comparison between traditional SMC and CRSMC. 

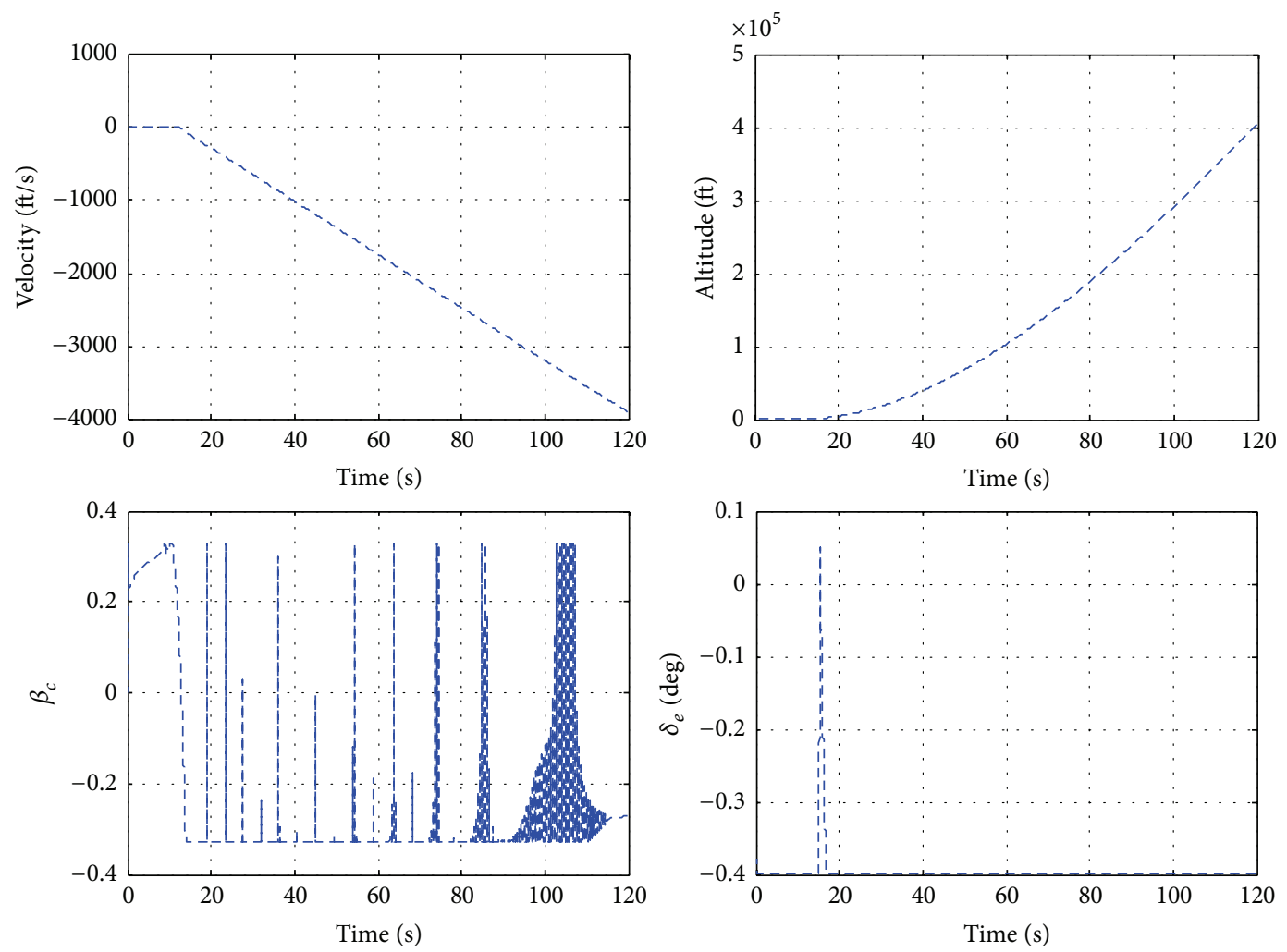

(a) Response for velocity step command
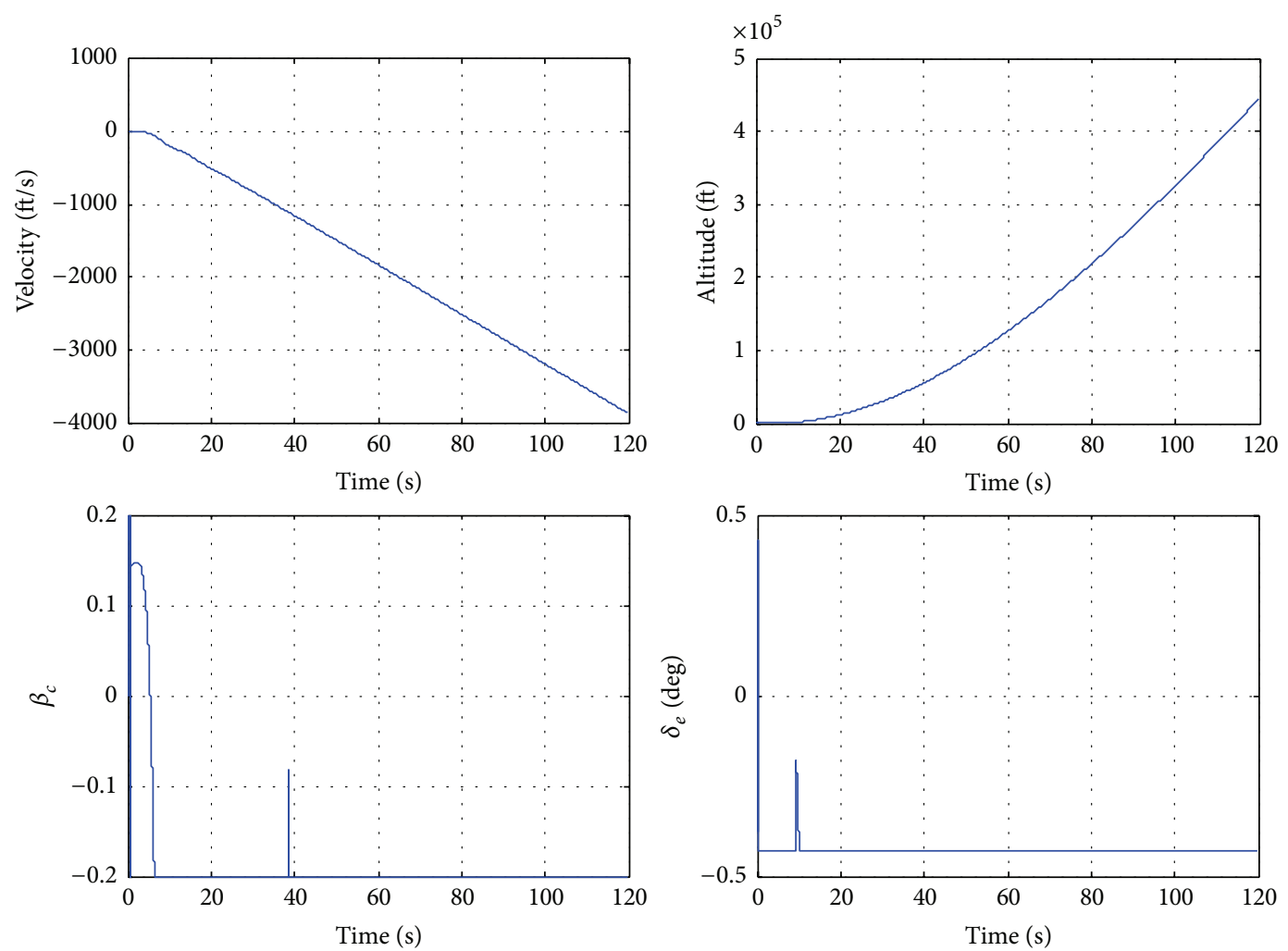

(b) Response for altitude step command

FIGURE 8: Step response results when actuator saturation occurs. 

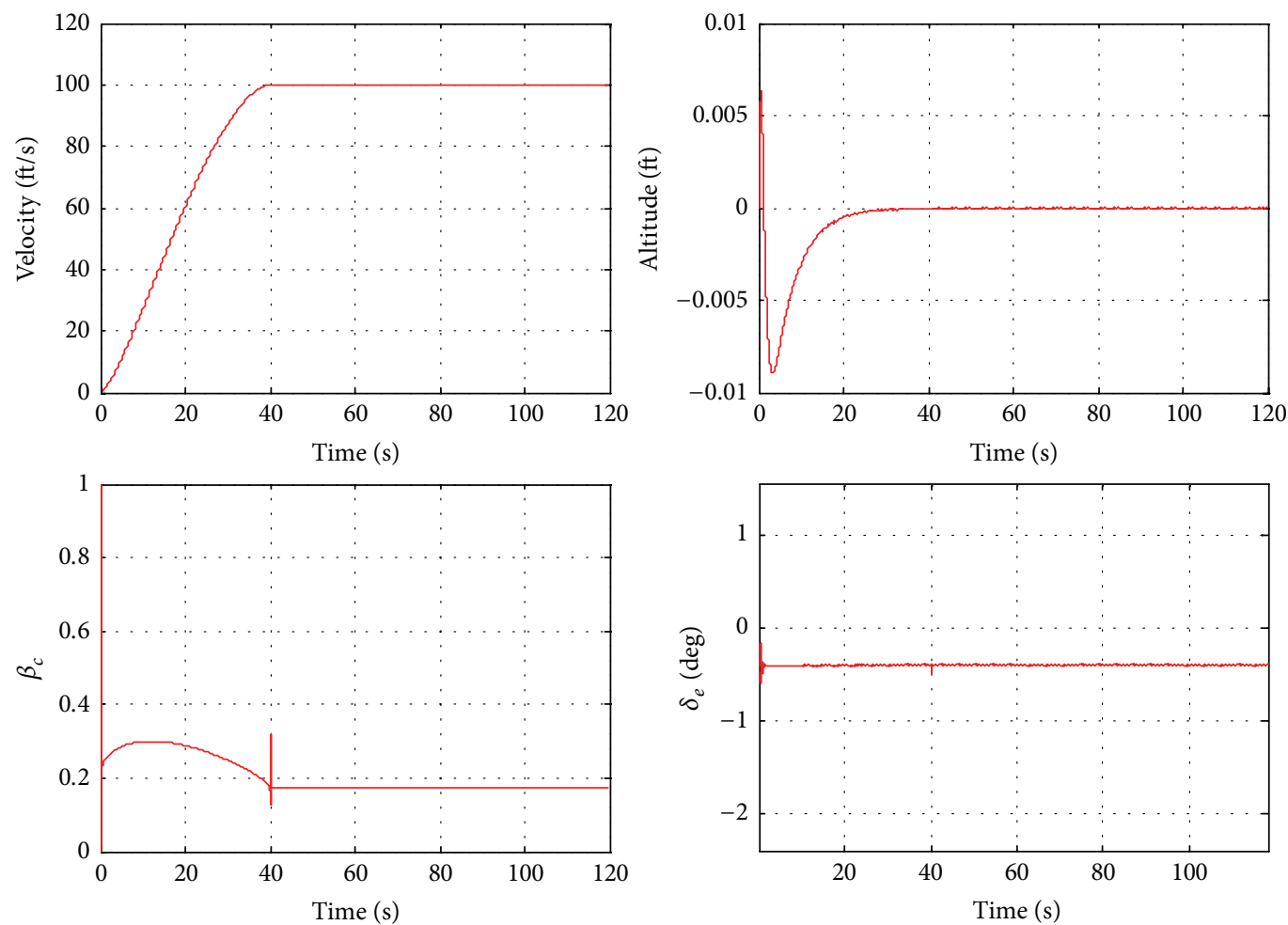

(a) Response for velocity step command
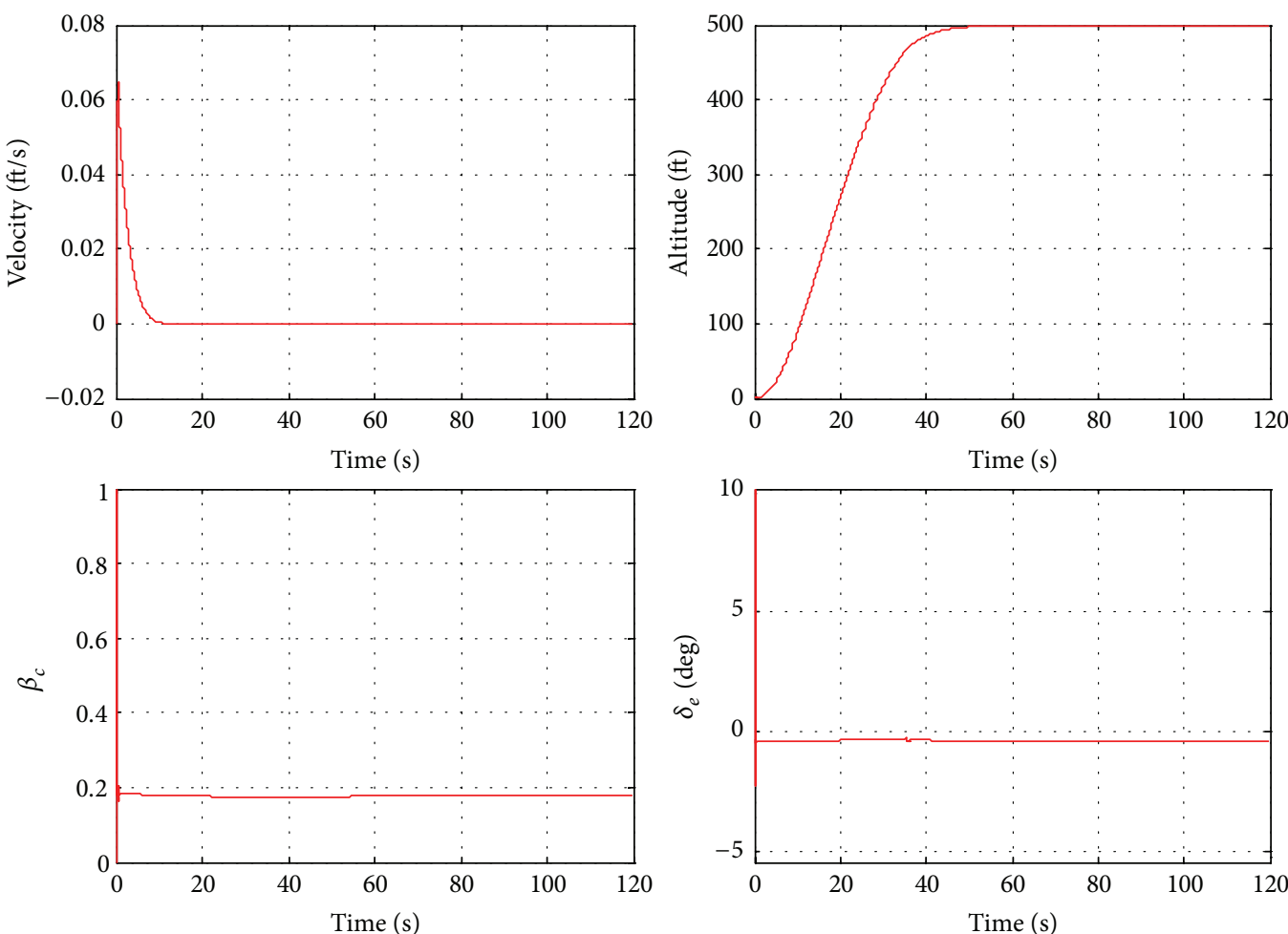

(b) Response for altitude step command

Figure 9: Step response under antiwindup controller. 


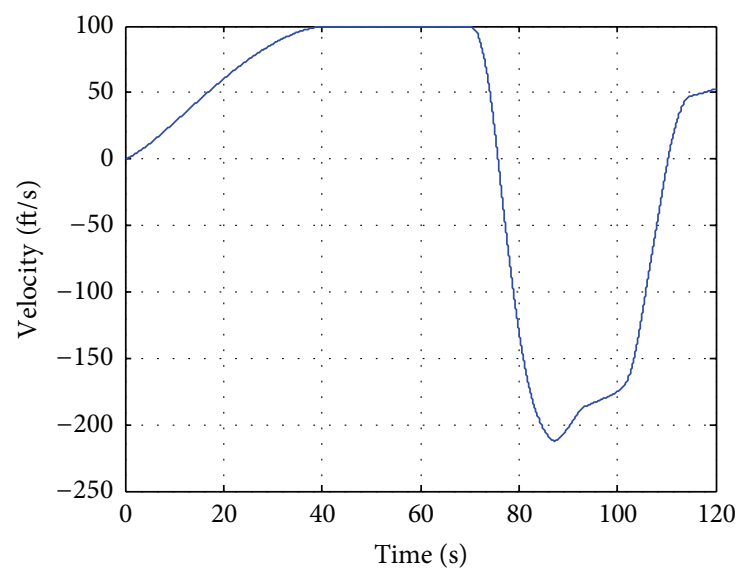

(a) Velocity

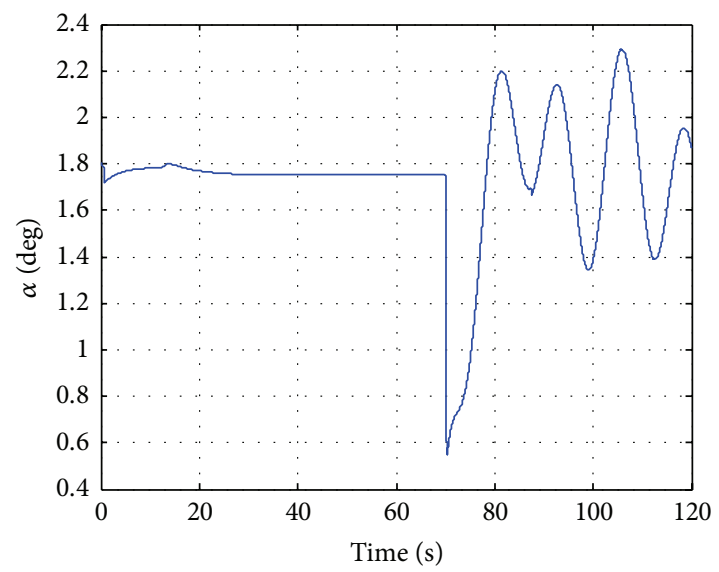

(c) Angle of attack

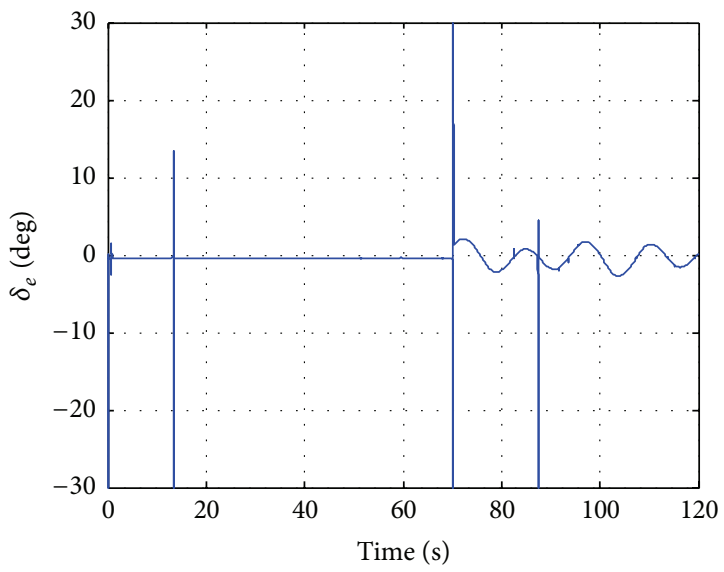

(e) Elevator deflection angle

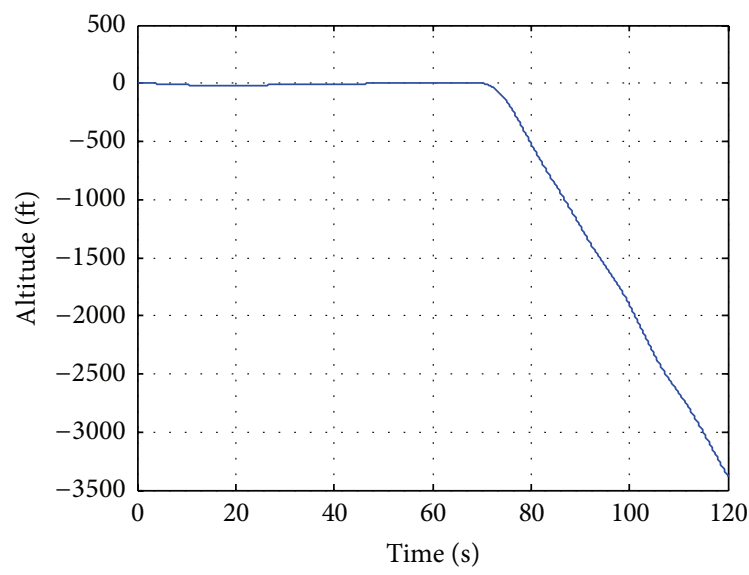

(b) Altitude

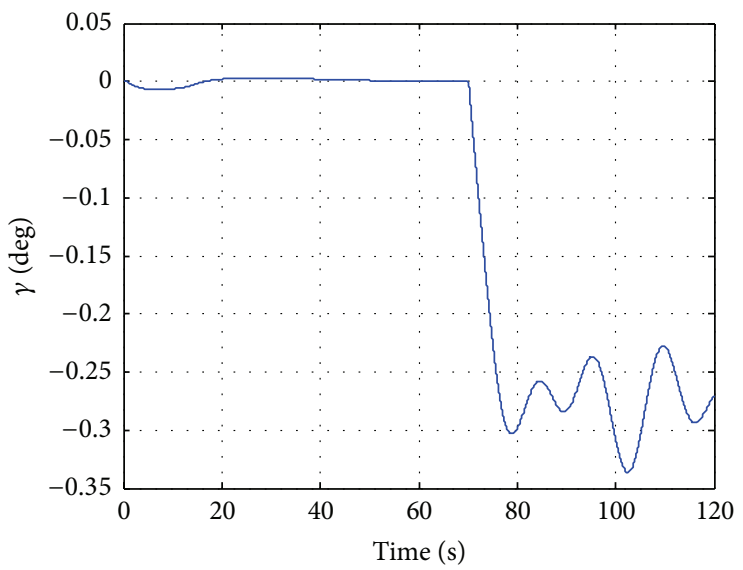

(d) Flight path angle

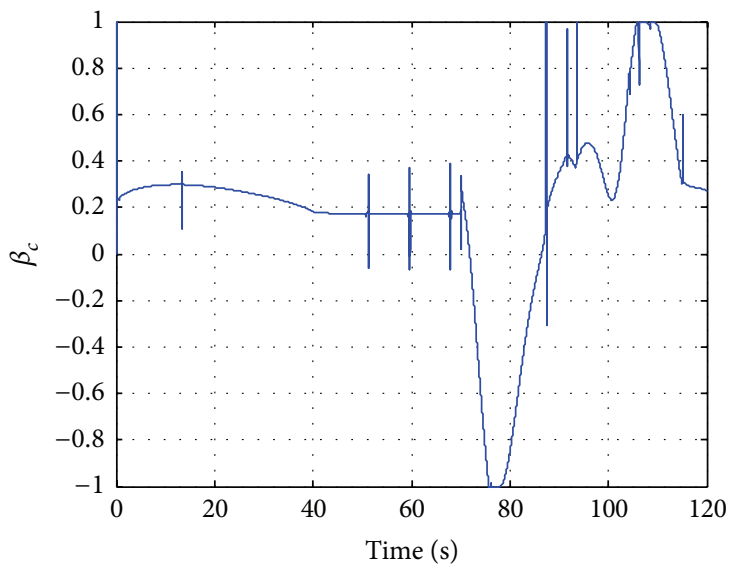

(f) Throttle setting

FIGURE 10: Response for velocity step command with disturbance.

By means of comparing, the proposed CRSMC achieves steady state faster than the traditional SMC. In addition, the altitude variable does not converge to zero in Figure 7(a) under the traditional SMC. In particular, the actuator response curves of SMC have large chattering, while the CRSMC do not.
When the actuator saturation occurs and controller (19) is taken, the simulation results for velocity step command and altitude step command are shown in Figure 8.

In Figure 8, it is obviously seen that the system is divergent when the actuator saturation occurs, if controller (19) is adopted. At this time, the HFV will lose control, which should 


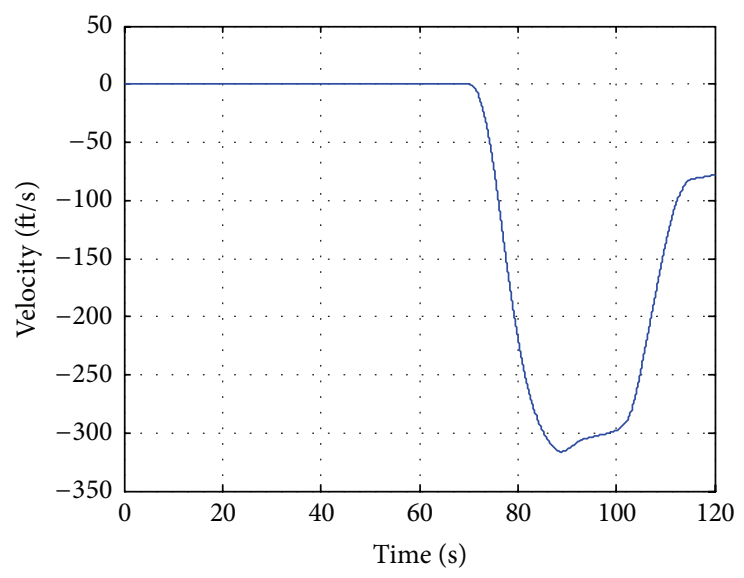

(a) Velocity

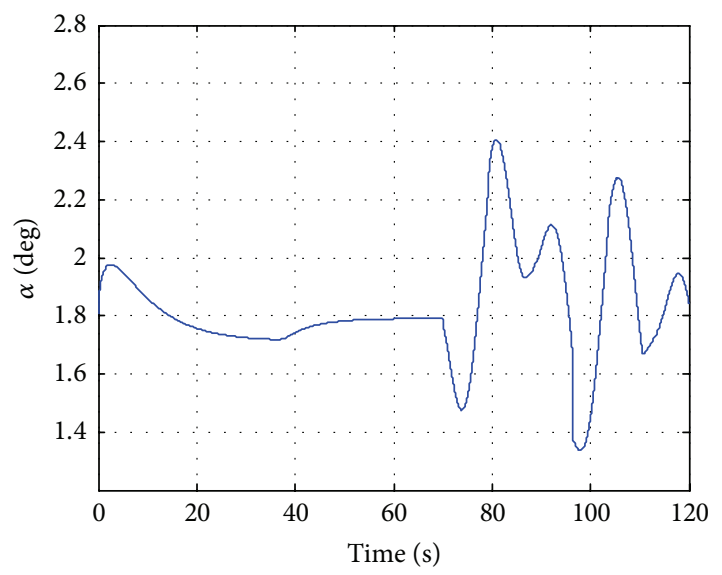

(c) Angle of attack

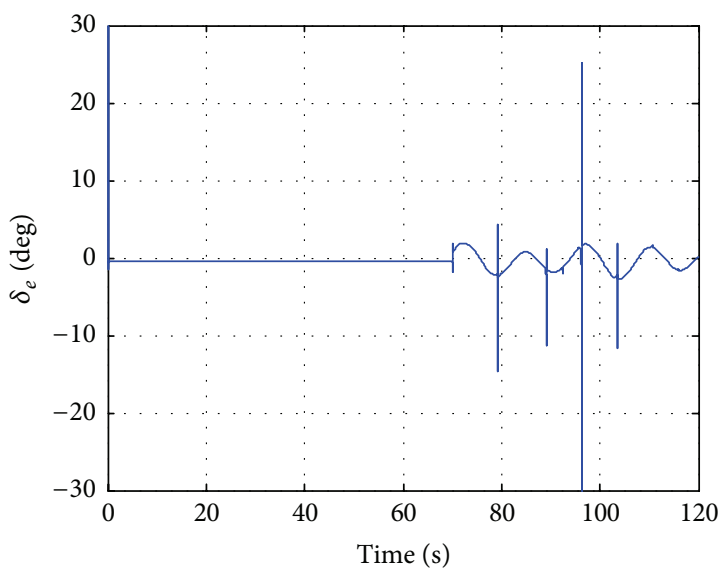

(e) Elevator deflection angle

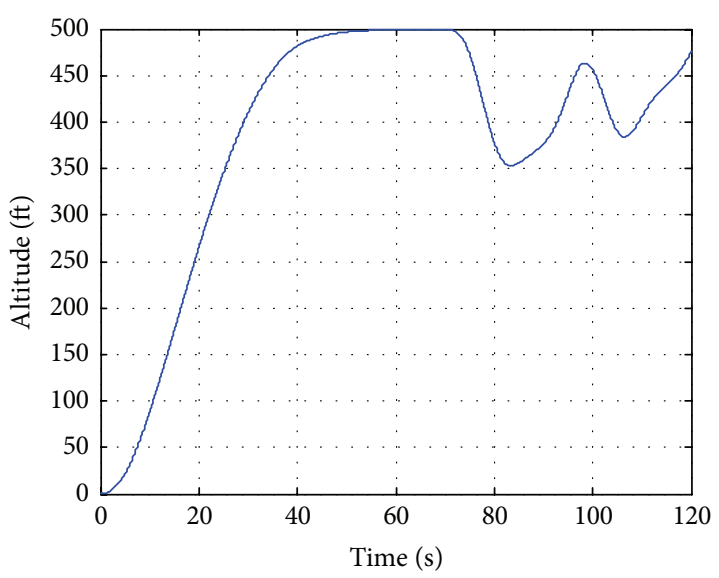

(b) Altitude

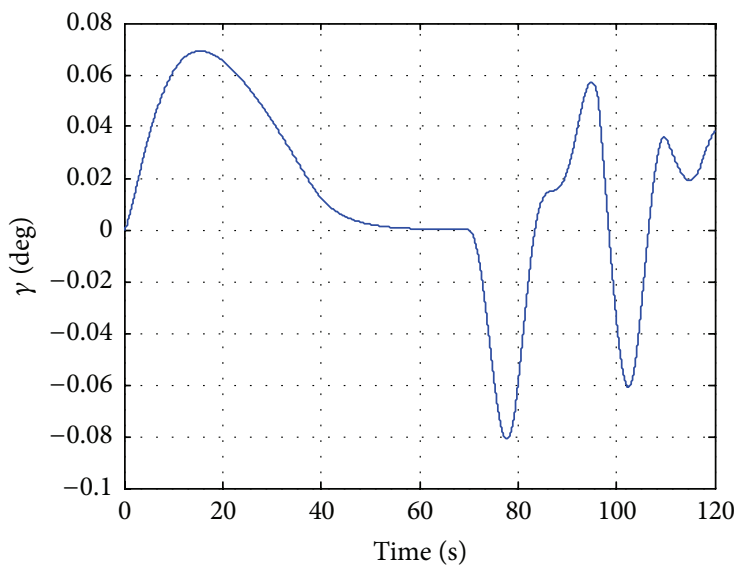

(d) Flight path angle

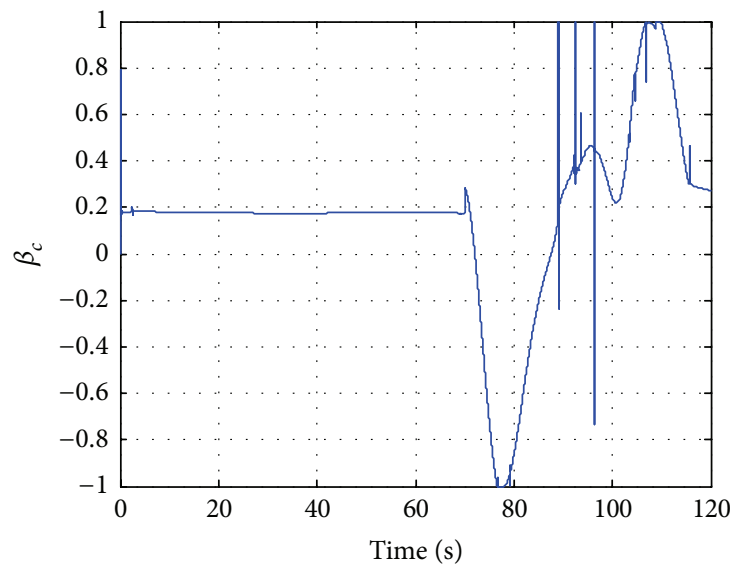

(f) Throttle setting

FIGURE 11: Response for altitude command with disturbance.

be avoided. When the antiwindup controller is taken up, the simulation results are shown in Figure 9.

In Figure 9, the system becomes stable and converges when the antiwindup controller is adopted. The simulation results in Figure 9 prove the effectiveness of the antiwindup controller of this paper.
When the external disturbances in (87) are considered, the system responses under CRSMC are shown in Figures 10 and 11 .

Because the external disturbances are added at 70th second, each parameter curve starts to shake after $70 \mathrm{~s}$, even becoming unstable in Figures 10 and 11. Thus, it is seen that 


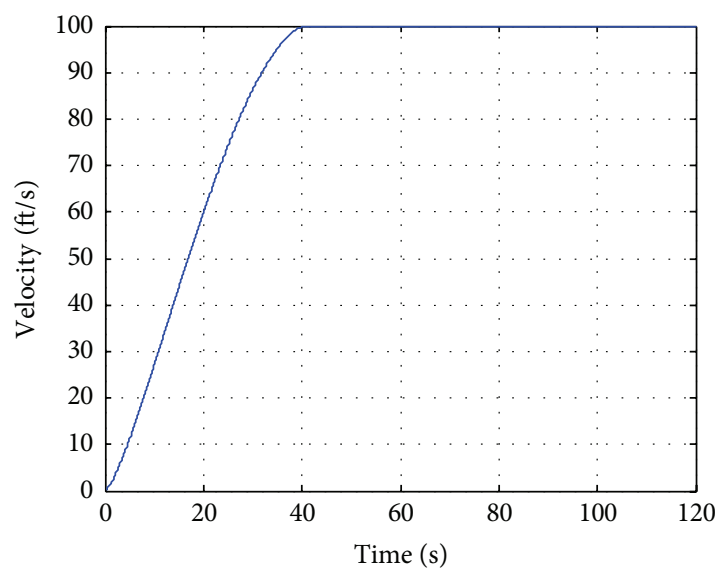

(a) Velocity

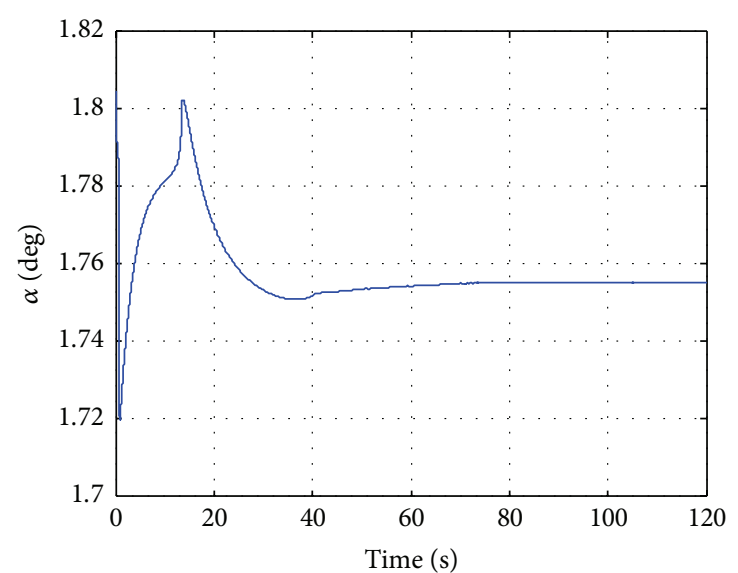

(c) Angle of attack

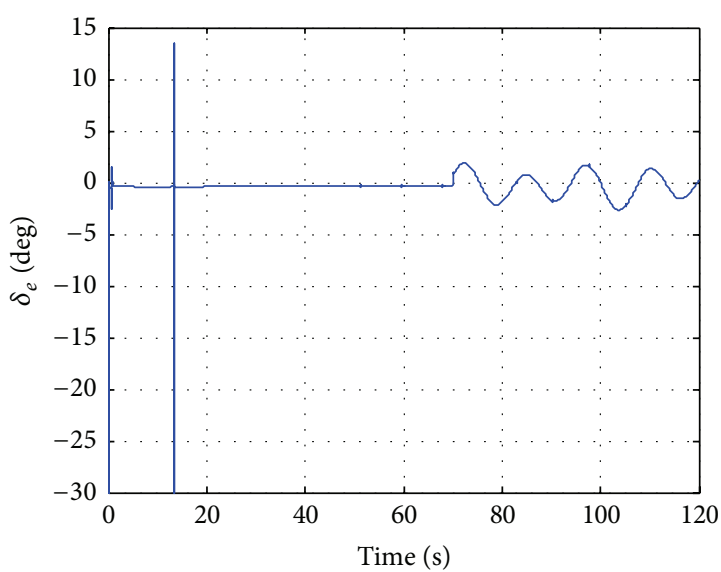

(e) Elevator deflection angle

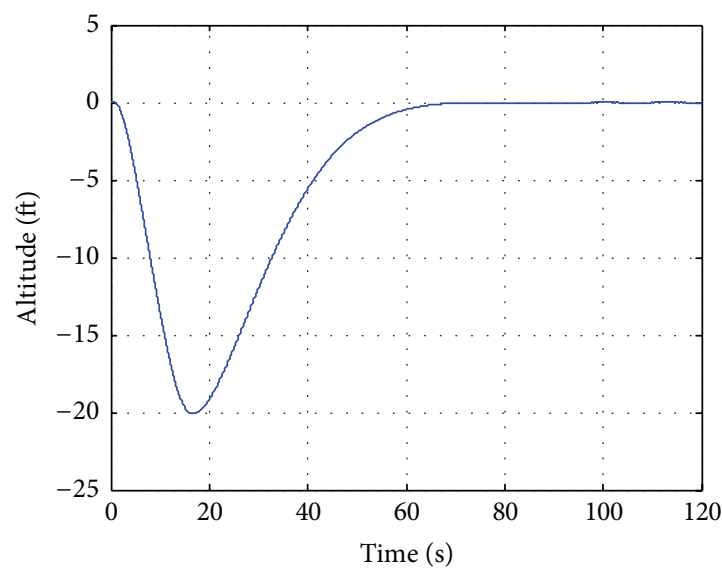

(b) Altitude

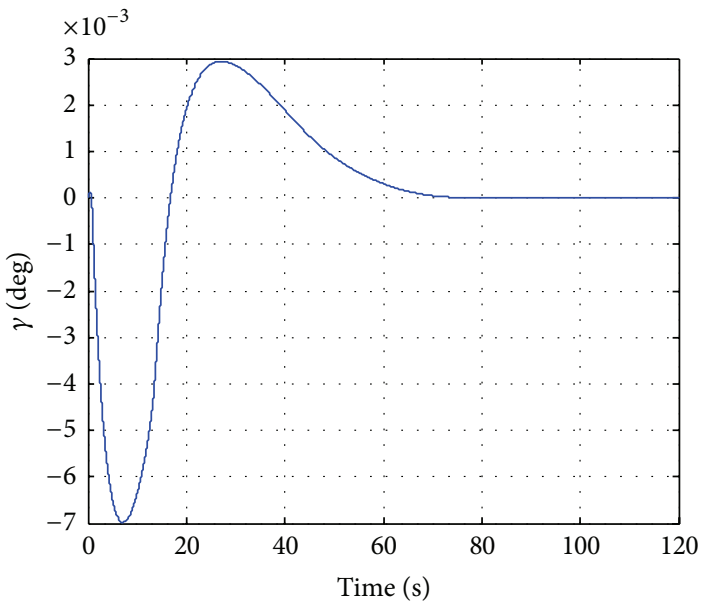

(d) Flight path angle

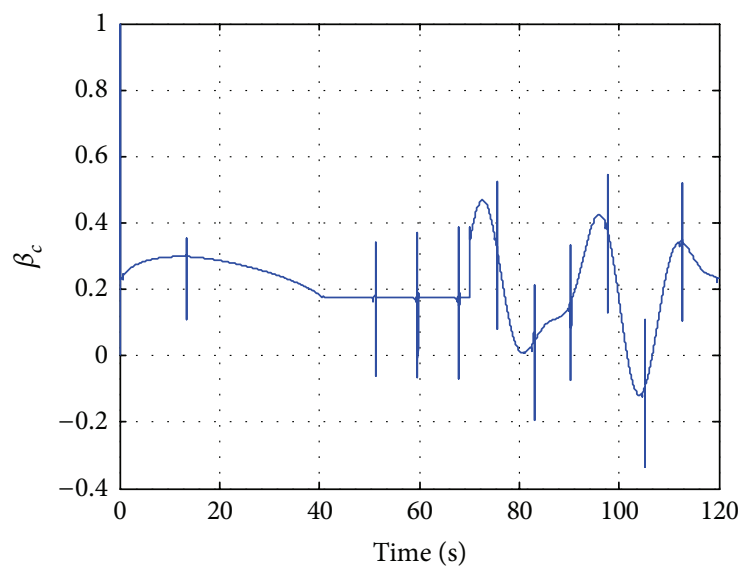

(f) Throttle setting

FIGURE 12: Response for velocity step command with IEDO.

the disturbances seriously affect the system, which must be suppressed.

When the IEDO is applied to estimate the disturbance and to compensate in CRSMC, the simulation results are shown in Figures 12 and 13.
Comparing Figures 10(a)-10(d) and 12(a)-12(d) and Figures $11(\mathrm{a})-11(\mathrm{~d})$ and $13(\mathrm{a})-13(\mathrm{~d})$, it is obviously seen that the responses with IEDO recover to the condition without disturbance; namely, the disturbances are well suppressed. In Figure 13(a), little shaking appears after the disturbance 


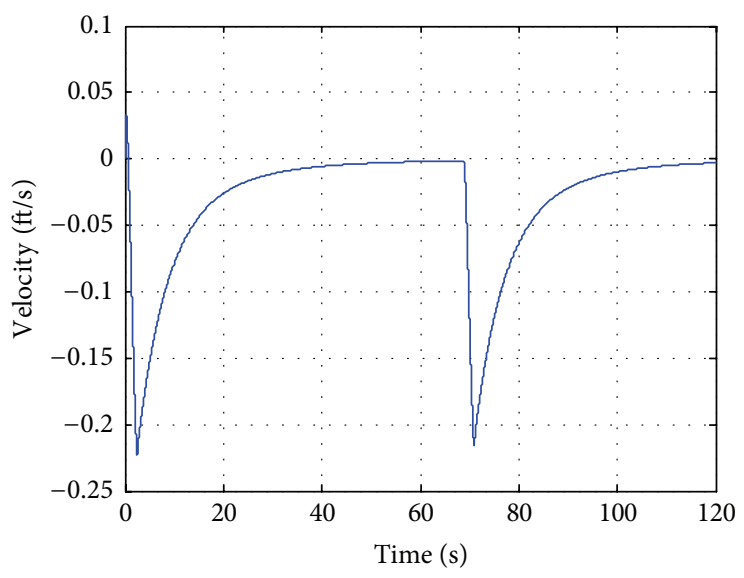

(a) Velocity

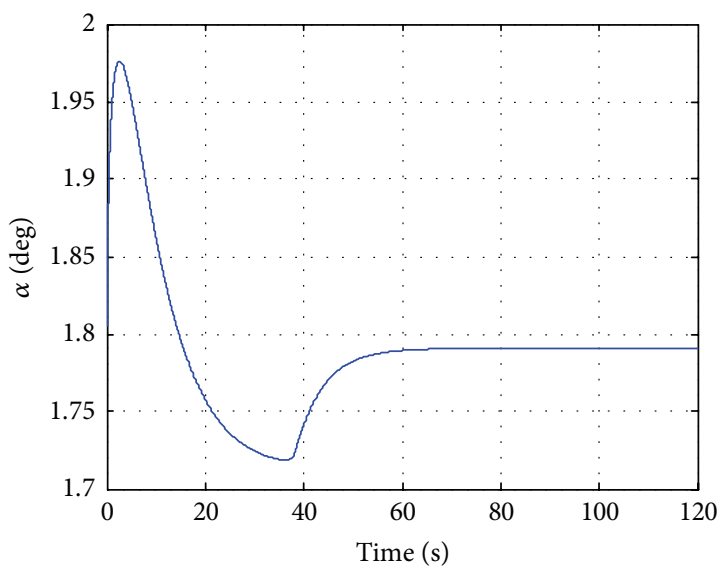

(c) Angle of attack

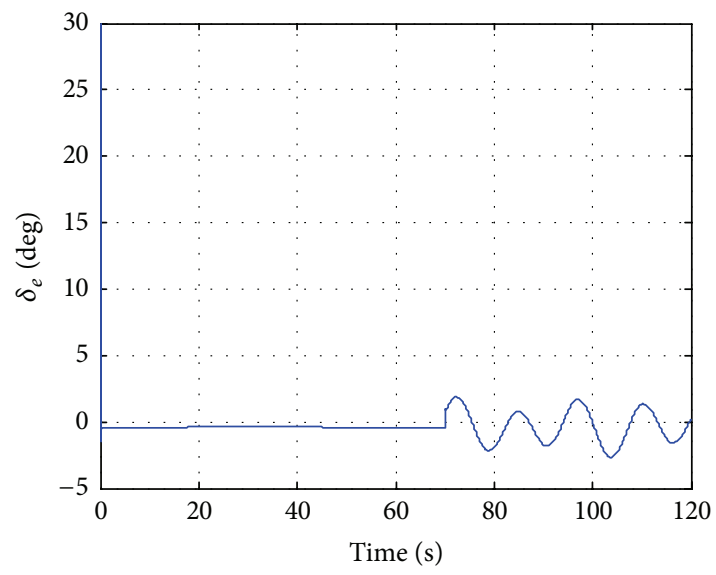

(e) Elevator deflection angle

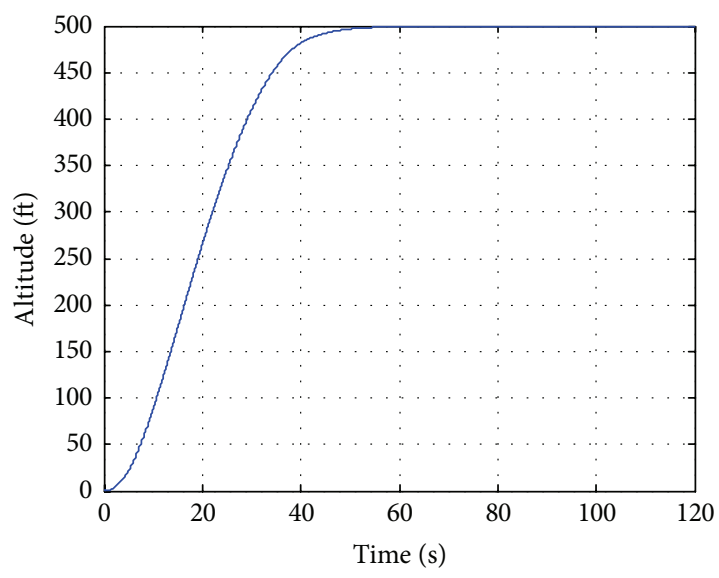

(b) Altitude

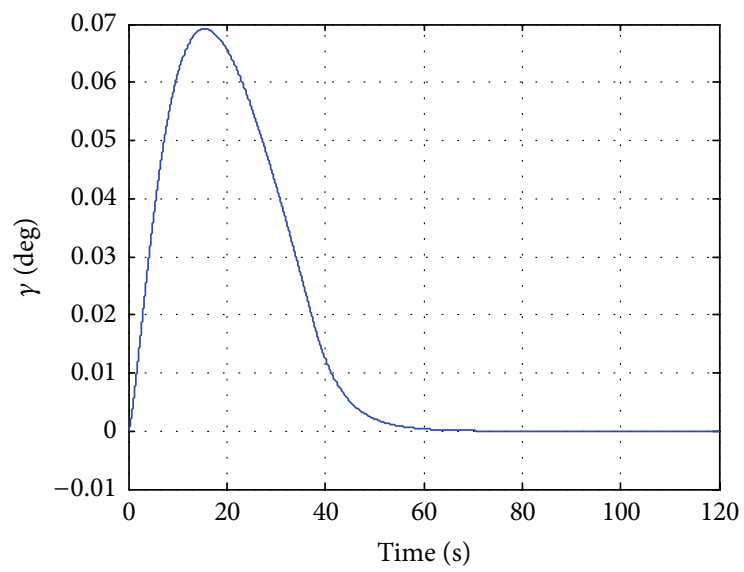

(d) Flight path angle

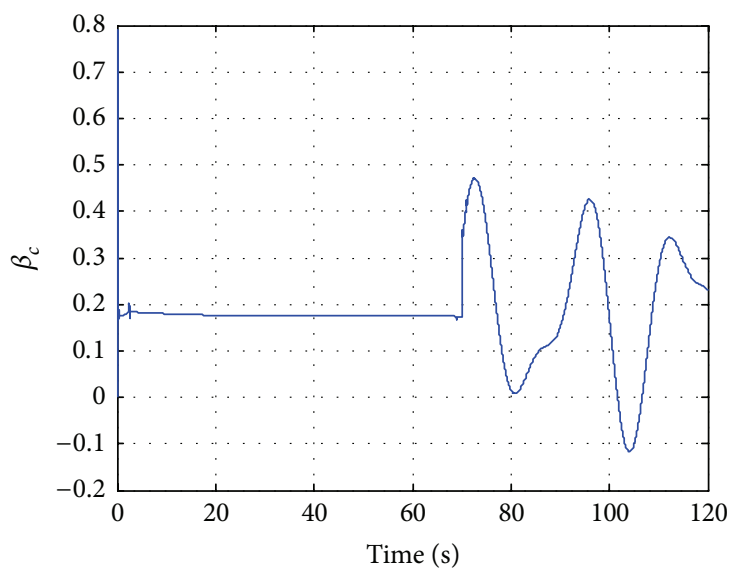

(f) Throttle setting

FIGURE 13: Response for altitude step command with IEDO.

is added to the system. However, it disappears soon and the curves converge to stable condition again. The estimated values by IEDO are regarded as compensation in controller, which is seen in Figures 12(e), 12(f), 13(e), and 13(f).

The estimation results for external disturbances are shown in Figure 14.
It is seen from Figure 14 that the disturbances are evaluated accurately, which manifests the notion that the IEDO has perfect performance.

To further illustrate the effectiveness of the method in this paper on interference suppression, two other kinds of disturbance are added to simulation for comparison; that is, 

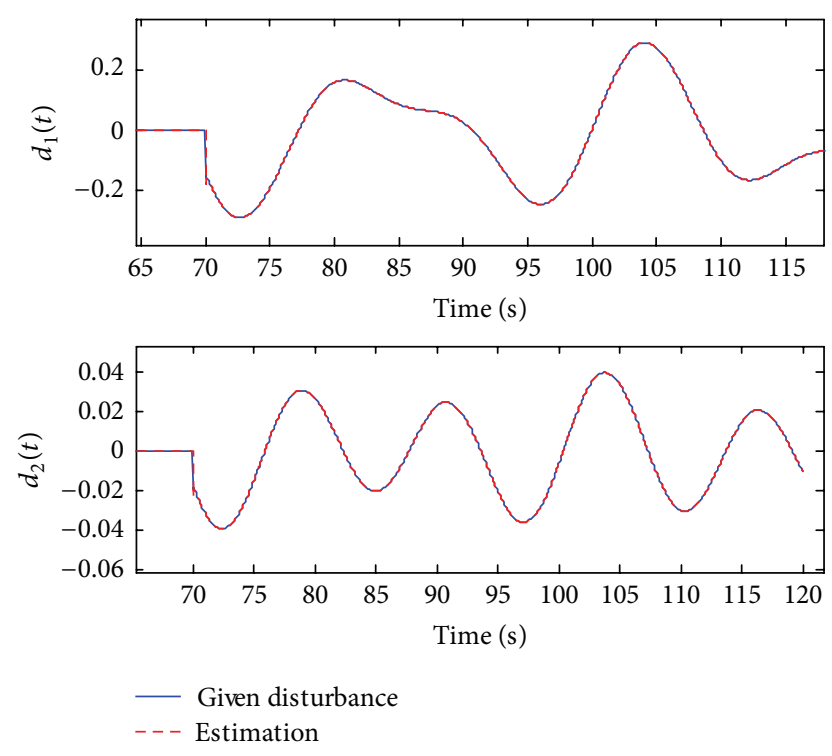

FIGURE 14: Disturbance estimation results.

$$
\begin{aligned}
& d_{1}(t)=0.15 \sin (0.3 t)+0.2 \cos (0.4 t), \\
& d_{2}(t)=0.02 \sin (0.4 t)+0.02 \cos (0.4 t), \\
& d_{1}(t)=0.2 \sin (0.4 t)+0.15 \cos (0.5 t), \\
& d_{2}(t)=0.04 \sin (0.5 t)+0.03 \cos (0.2 t)
\end{aligned}
$$

Here, define the disturbance in (87) as "Situation 1," define the disturbance in (88) as "Situation 2," and define the disturbance in (89) as "Situation 3." Then, the comparison simulation results between the three situations are shown in Figure 15.

In Figure 15, it can be seen that the system keeps stable and convergent under each situation. According to every situation, the system response is similar and the system state curves are consistent. The disturbance estimation errors of

$$
\begin{aligned}
& \omega_{21}=\left[\begin{array}{c}
\left(\frac{\partial^{2} T}{\partial V^{2}}\right) \cos \alpha-\frac{\partial^{2} D}{\partial V^{2}} \\
0 \\
-\left(\frac{\partial T}{\partial V}\right) \sin \alpha-\frac{\partial^{2} D}{\partial V \partial \alpha} \\
\left(\frac{\partial^{2} T}{\partial V \partial \beta}\right) \cos \alpha \\
0
\end{array}\right], \\
& \boldsymbol{\omega}_{22}=\left[\begin{array}{c}
0 \\
\frac{m \mu \sin \gamma}{r^{2}} \\
0 \\
0 \\
\frac{2 m \mu \cos \gamma}{r^{3}}
\end{array}\right] \text {, }
\end{aligned}
$$

three situations in Figure 15(c) are less than $1 \times 10^{-5}$, which is small enough for the estimation. The comparison between the three situations proves the practicability of the proposed method in the paper.

By means of the above simulations, the proposed scheme has perfect tracking and disturbance rejection performance for longitudinal dynamics of FHV under control constraints. In addition, the presented approach is robust for multiple uncertainties. Meanwhile, it has no chattering phenomenon. The scheme of CRSMC with antiwindup controller plus IEDO possesses favorable performance.

\section{Conclusions}

The continuous recursive sliding mode controller (CRSMC) with improved extended disturbance observer (IEDO) is presented for longitudinal dynamics of generic hypersonic flight vehicle in the presence of multiple uncertainties under control constraints in this paper. For each channel, a sliding mode tracking controller is designed based on a set of recursive sliding mode manifolds, which are nonsingular. The controller is continuous and has no chattering problem. For the actuator saturation problem, a novel compensation factor is designed for compensating the CRSMC. In response to external disturbance, an improved disturbance observer based on extended disturbance observer (EDO) is presented. The composite approach is CRSMC plus IEDO, in which the estimated value by IEDO is regarded as compensation value in CRSMC. Numerical simulation for cruise flight condition of HFV is conducted. Simulation results have confirmed effectiveness and availability of the proposed scheme.

\section{Appendix}

The detailed expressions of the vectors $\boldsymbol{\Omega}_{1}, \boldsymbol{\Pi}_{1}$ and matrices $\Omega_{2}, \Pi_{2}$ are as follows:

$$
\boldsymbol{\Omega}_{2}=\left[\begin{array}{lllll}
\boldsymbol{\omega}_{21} & \boldsymbol{\omega}_{22} & \boldsymbol{\omega}_{23} & \omega_{24} & \omega_{25}
\end{array}\right],
$$

where 

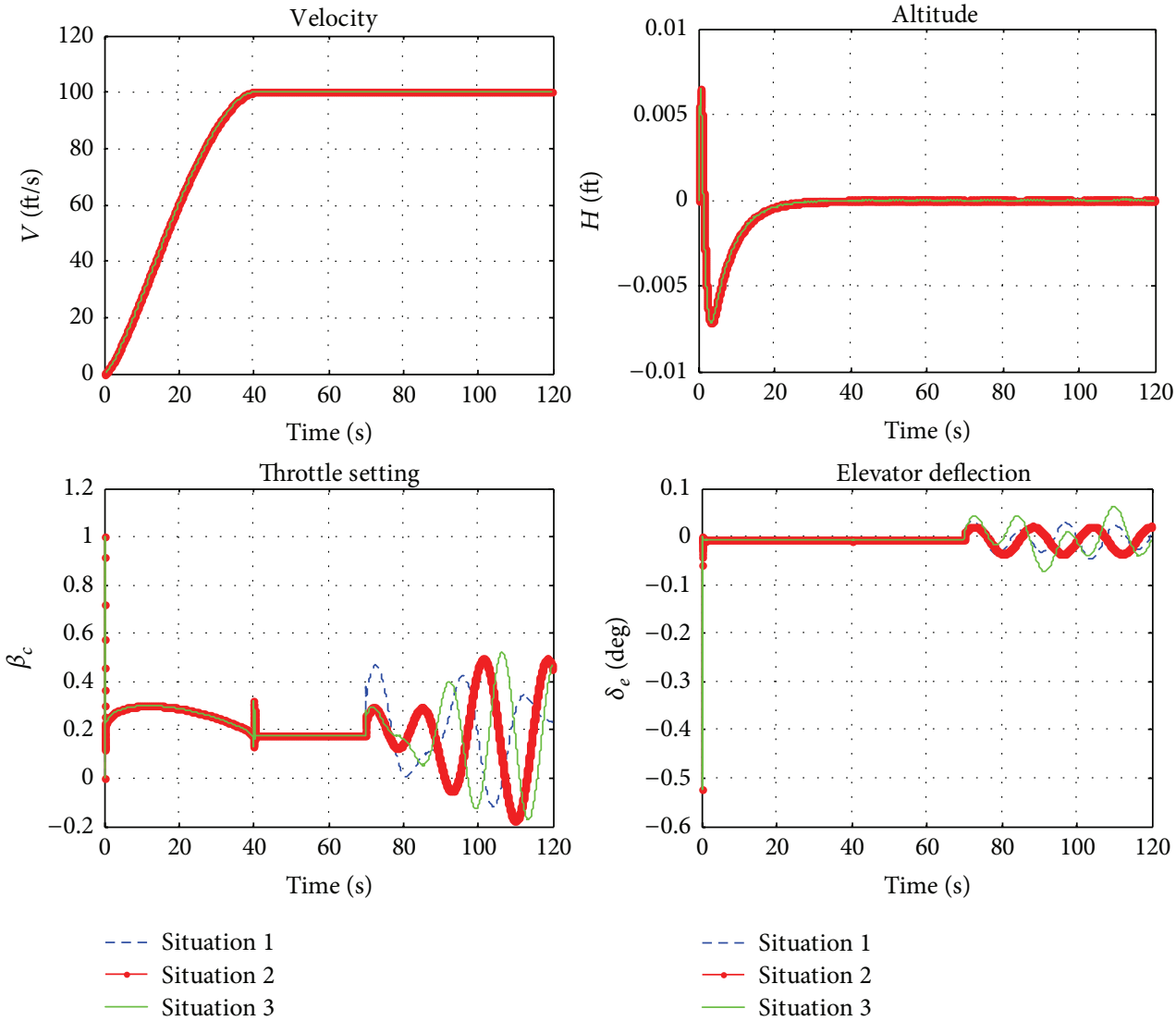

- - Situation 1

$\longrightarrow$ Situation 2

- Situation 3

(a) Comparison for velocity step command response
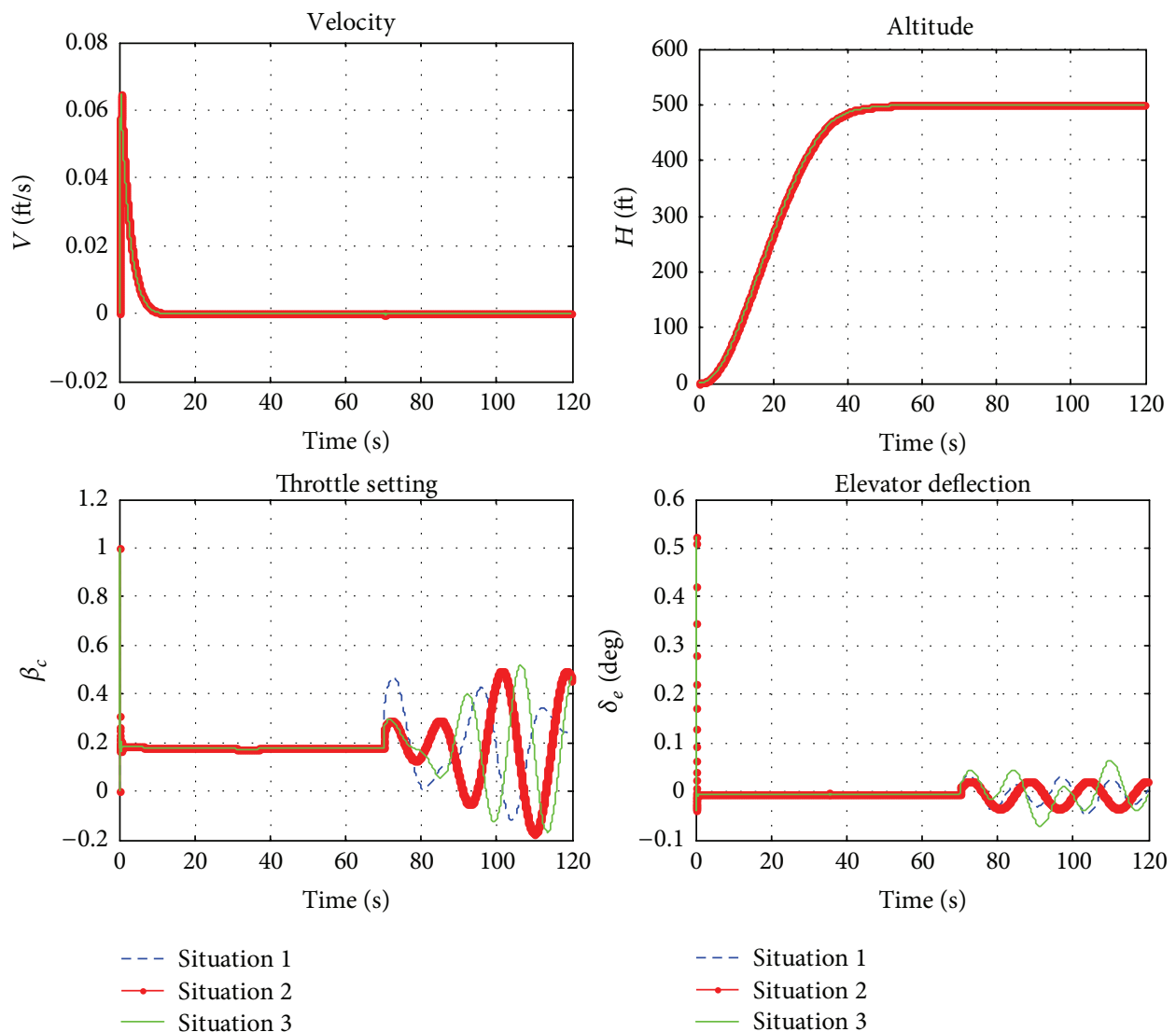

Situatior

(b) Comparison for altitude step command response

FIgure 15: Continued. 

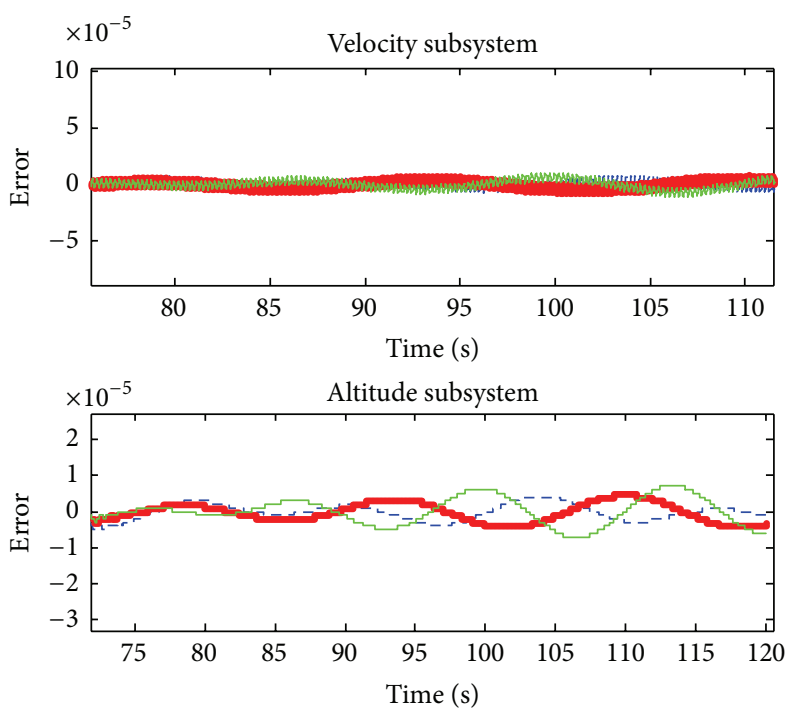

- - Situation 1

$\rightarrow$ Situation 2

- Situation 3

(c) Error comparison

FIgURE 15: Step response of three situations.

$$
\begin{aligned}
& \boldsymbol{\omega}_{23}=\left[\begin{array}{c}
-\left(\frac{\partial T}{\partial V}\right) \sin \alpha-\left(\frac{\partial^{2} D}{\partial V \partial \alpha}\right) \\
0 \\
-T \cos \alpha-\left(\frac{\partial^{2} D}{\partial \alpha^{2}}\right) \\
-\left(\frac{\partial T}{\partial \beta}\right) \sin \alpha \\
0
\end{array}\right], \\
& \boldsymbol{\omega}_{24}=\left[\begin{array}{c}
\frac{\partial^{2} T}{\partial V \partial \beta} \cos \alpha \\
0 \\
-\left(\frac{\partial T}{\partial \beta}\right) \sin \alpha \\
0 \\
0
\end{array}\right], \\
& \boldsymbol{\omega}_{25}=\left[\begin{array}{c}
0 \\
\frac{2 m \mu \cos \gamma}{r^{3}} \\
0 \\
0 \\
-\frac{6 m \mu \sin \gamma}{r^{4}}
\end{array}\right] \text {, }
\end{aligned}
$$




$$
\begin{aligned}
& \Pi_{1}=\left[\begin{array}{c}
\frac{\partial L / \partial V+(\partial T / \partial V) \sin \alpha}{m V}-\frac{L+T \sin \alpha}{m V^{2}}+\frac{\mu \cos \gamma}{V^{2} r^{2}}+\frac{\cos \gamma}{r} \\
\frac{\mu \sin \gamma}{V r^{2}}-\frac{V \sin \gamma}{r} \\
\frac{\partial L / \partial \alpha+T \cos \alpha}{m V} \\
\frac{(\partial T / \partial \beta) \sin \alpha}{m V} \\
\frac{2 \mu \cos \gamma}{V r^{3}}-\frac{V \cos \gamma}{r^{2}}
\end{array}\right] \\
& \mathbf{\Omega}_{1}=\left[\begin{array}{c}
\left(\frac{\partial T}{\partial V}\right) \cos \alpha-\frac{\partial D}{\partial V} \\
-\frac{m \mu \cos \gamma}{r^{2}} \\
-T \sin \alpha-\left(\frac{\partial D}{\partial \alpha}\right) \\
\left(\frac{\partial T}{\partial \beta}\right) \cos \alpha \\
\frac{2 m \mu \sin \gamma}{r^{3}}
\end{array}\right]
\end{aligned}
$$$$
\Pi_{2}=\left[\begin{array}{lllll}
\pi_{21} & \pi_{22} & \pi_{23} & \pi_{24} & \pi_{25}
\end{array}\right],
$$$$
\boldsymbol{\pi}_{21}=\left[\begin{array}{c}
\frac{\partial^{2} L / \partial V^{2}+\left(\partial^{2} T / \partial V^{2}\right) \sin \alpha}{m V}-\frac{2[\partial L / \partial V+(\partial T / \partial V) \sin \alpha]}{m V^{2}}+\frac{2(L+T \sin \alpha)}{m V^{3}}-\frac{2 \mu \cos \gamma}{V^{3} r^{2}} \\
-\frac{\mu \sin \gamma}{V^{2} r^{2}}-\frac{\sin \gamma}{r} \\
\frac{\left(\partial^{2} L / \partial \alpha \partial V\right)+(\partial T / \partial V) \cos \alpha}{m V}-\frac{\partial L / \partial \alpha+T \cos \alpha}{m V^{2}} \\
\frac{\left(\partial^{2} T / \partial \beta \partial V\right) \sin \alpha}{m V}-\frac{(\partial T / \partial \beta) \sin \alpha}{m V^{2}} \\
-\frac{2 \mu \cos \gamma}{V^{2} r^{3}}-\frac{\cos \gamma}{r^{2}}
\end{array}\right],
$$$$
\pi_{22}=\left[\begin{array}{c}
-\frac{\mu \sin \gamma}{V^{2} r^{2}}-\frac{\sin \gamma}{r} \\
\frac{\mu \cos \gamma}{V r^{2}}-\frac{V \cos \gamma}{r} \\
0 \\
0 \\
-\frac{2 \mu \sin \gamma}{V r^{3}}+\frac{V \sin \gamma}{r^{2}}
\end{array}\right],
$$ 


$$
\begin{aligned}
& \boldsymbol{\pi}_{23}=\left[\begin{array}{c}
\frac{\left(\partial^{2} L / \partial V \partial \alpha\right)+(\partial T / \partial V) \cos \alpha}{m V}-\frac{\partial L / \partial \alpha+T \cos \alpha}{m V^{2}} \\
0 \\
\frac{\partial^{2} L / \partial \alpha^{2}-T \sin \alpha}{m V} \\
\frac{(\partial T / \partial \beta) \cos \alpha}{m V} \\
0
\end{array}\right], \\
& \pi_{24}=\left[\begin{array}{c}
\frac{\left(\partial^{2} T / \partial V \partial \beta\right) \sin \alpha}{m V}-\frac{(\partial T / \partial \beta) \sin \alpha}{m V^{2}} \\
0 \\
\frac{(\partial T / \partial \beta) \cos \alpha}{m V} \\
0 \\
0
\end{array}\right], \\
& \pi_{25}=\left[\begin{array}{c}
-\frac{2 \mu \cos \gamma}{V^{2} r^{3}}-\frac{\cos \gamma}{r^{2}} \\
-\frac{2 \mu \sin \gamma}{V r^{3}}+\frac{V \sin \gamma}{r^{2}} \\
0 \\
0 \\
-\frac{6 \mu \cos \gamma}{V r^{4}}+\frac{2 V \cos \gamma}{r^{3}}
\end{array}\right] .
\end{aligned}
$$

\section{Conflict of Interests}

The authors declare that there is no conflict of interests regarding the publication of this paper.

\section{Acknowledgment}

This work is supported by the National Natural Science Foundation of China (91216304).

\section{References}

[1] F. R. Chavez and D. K. Schmidt, "Analytical aeropropulsive/aeroelastic hypersonic-vehicle model with dynamic analysis," Journal of Guidance, Control, and Dynamics, vol. 17, no. 6, pp. 13081319, 1994.

[2] M. A. Bolender and D. B. Doman, A Non-Linear Model for the Longitudinal Dynamics of a Hypersonic Air-Breathing Vehicle, Defense Technical Information Center, 2006.

[3] M. A. Bolender and D. B. Doman, "Nonlinear longitudinal dynamical model of an air-breathing hypersonic vehicle," Journal of Spacecraft and Rockets, vol. 44, no. 2, pp. 374-387, 2007.

[4] C. I. Marrison and R. F. Stengel, "Design of robust control systems for a hypersonic aircraft," Journal of Guidance, Control, and Dynamics, vol. 21, no. 1, pp. 58-63, 1998.

[5] Q. Wang and R. F. Stengel, "Robust nonlinear control of a hypersonic aircraft," Journal of Guidance, Control, and Dynamics, vol. 23, no. 4, pp. 577-585, 2000.

[6] J. T. Parker, M. A. Bolender, D. B. Doman, A. Serrani, and S. Yurkovich, "Control-oriented modeling of an air-breathing hypersonic vehicle," Journal of Guidance, Control, and Dynamics, vol. 30, no. 3, pp. 856-869, 2007.

[7] O. U. Rehman, B. Fidan, and I. R. Petersen, "Uncertainty modeling and robust minimax LQR control of multivariable nonlinear systems with application to hypersonic flight," Asian Journal of Control, vol. 14, no. 5, pp. 1180-1193, 2012.

[8] O. Ur Rehman, I. R. Petersen, and B. Fidan, "Feedback linearization-based robust nonlinear control design for hypersonic flight vehicles," Proceedings of the Institution of Mechanical Engineers-Part I, vol. 227, no. 1, pp. 3-11, 2013.

[9] O. U. Rehman, I. R. Petersen, and B. Fidan, "Robust nonlinear control design of a hypersonic flight vehicle using minimax 
linear quadratic gaussian control," in Proceedings of the 49th IEEE Conference on Decision and Control (CDC '10), pp. 62196224, IEEE, Atlanta, Ga, USA, December 2010.

[10] R. Qi, Y. Huang, B. Jiang, and G. Tao, "Adaptive backstepping control for a hypersonic vehicle with uncertain parameters and actuator faults," Proceedings of the Institution of Mechanical Engineers-Part I, vol. 227, no. 1, pp. 51-61, 2013.

[11] W. A. Butt, L. Yan, and A. S. Kendrick, "Adaptive dynamic surface control of a hypersonic flight vehicle with improved tracking," Asian Journal of Control, vol. 15, no. 2, pp. 594-605, 2013.

[12] H. Xu, M. D. Mirmirani, and P. A. Ioannou, "Adaptive sliding mode control design for a hypersonic flight vehicle," Journal of Guidance, Control, and Dynamics, vol. 27, no. 5, pp. 829-838, 2004.

[13] S. Yu, X. Yu, B. Shirinzadeh, and Z. Man, "Continuous finitetime control for robotic manipulators with terminal sliding mode," Automatica, vol. 41, no. 11, pp. 1957-1964, 2005.

[14] X. Yu and M. Zhihong, "Fast terminal sliding-mode control design for nonlinear dynamical systems," IEEE Transactions on Circuits and Systems I: Fundamental Theory and Applications, vol. 49, no. 2, pp. 261-264, 2002.

[15] Y. Feng, X. Yu, and Z. Man, "Non-singular terminal sliding mode control of rigid manipulators," Automatica, vol. 38, no. 12, pp. 2159-2167, 2002.

[16] Q. Zong, J. Wang, B. Tian, and Y. Tao, "Quasi-continuous highorder sliding mode controller and observer design for flexible hypersonic vehicle," Aerospace Science and Technology, vol. 27, no. 1, pp. 127-137, 2013.

[17] R. Zhang, C. Sun, J. Zhang, and Y. Zhou, "Second-order terminal sliding mode control for hypersonic vehicle in cruising flight with sliding mode disturbance observer," Journal of Control Theory and Applications, vol. 11, no. 2, pp. 299-305, 2013.

[18] M. Chen, S. S. Ge, and B. Ren, "Adaptive tracking control of uncertain MIMO nonlinear systems with input constraints," Automatica, vol. 47, no. 3, pp. 452-465, 2011.

[19] X. Wu and Z. Lin, "Dynamic anti-windup design in anticipation of actuator saturation," International Journal of Robust and Nonlinear Control, vol. 24, no. 2, pp. 295-312, 2014.

[20] G. A. Kiener, D. Lehmann, and K. H. Johansson, "Actuator saturation and anti-windup compensation in event-triggered control," Discrete Event Dynamic Systems, vol. 24, no. 2, pp. 173197, 2014.

[21] Y.-B. Liu, D.-B. Xiao, and Y.-P. Lu, "Research on advanced flight control methods based on actuator constraints for elastic model of hypersonic vehicle," Proceedings of the Institution of Mechanical Engineers, Part G: Journal of Aerospace Engineering, vol. 228, no. 9, pp. 1627-1637, 2014.

[22] J. Han, "The 'extended state observer' of a class of uncertain systems," Control and Decision, vol. 10, no. 1, pp. 85-88, 1995 (Chinese).

[23] G. Feng, Y.-F. Liu, and L. Huang, "A new robust algorithm to improve the dynamic performance on the speed control of induction motor drive," IEEE Transactions on Power Electronics, vol. 19, no. 6, pp. 1614-1627, 2004.

[24] Y. Xia, Z. Zhu, M. Fu, and S. Wang, "Attitude tracking of rigid spacecraft with bounded disturbances," IEEE Transactions on Industrial Electronics, vol. 58, no. 2, pp. 647-659, 2011.

[25] J. Su, H. Ma, W. Qiu, and Y. Xi, “Task-independent robotic uncalibrated hand-eye coordination based on the extended state observer," IEEE Transactions on Systems, Man, and Cybernetics, Part B: Cybernetics, vol. 34, no. 4, pp. 1917-1922, 2004.
[26] T. Yu, K. W. Chan, J. P. Tong, B. Zhou, and D. H. Li, "Coordinated robust nonlinear boiler-turbine-generator control systems via approximate dynamic feedback linearization," Journal of Process Control, vol. 20, no. 4, pp. 365-374, 2010.

[27] Z. Gao, "Scaling and bandwidth-parameterization based controller tuning," in Proceedings of the American Control Conference, vol. 6, pp. 4989-4996, June 2003.

[28] D. Ginoya, P. D. Shendge, and S. B. Phadke, "Sliding mode control for mismatched uncertain systems using an extended disturbance observer," IEEE Transactions on Industrial Electronics, vol. 61, no. 4, pp. 1983-1992, 2014.

[29] W.-H. Chen, D. J. Ballance, P. J. Gawthrop, and J. O’Reilly, “A nonlinear disturbance observer for robotic manipulators," IEEE Transactions on Industrial Electronics, vol. 47, no. 4, pp. 932-938, 2000.

[30] L. Guo and W.-H. Chen, "Disturbance attenuation and rejection for systems with nonlinearity via DOBC approach," International Journal of Robust and Nonlinear Control, vol. 15, no. 3, pp. 109-125, 2005.

[31] J. Yang, S. Li, and W.-H. Chen, "Nonlinear disturbance observer-based control for multi-input multi-output nonlinear systems subject to mismatching condition," International Journal of Control, vol. 85, no. 8, pp. 1071-1082, 2012.

[32] S. Yu, J. Yu, X. Yu, and H. Xu, "A novel recursive terminal sliding mode with finite-time convergence," in Proceedings of the 17th IFAC World Congress, pp. 5945-5949, International Federation of Automatic Control, Seoul, Republic of Korea, July 2008.

[33] Z. Gao, "Active disturbance rejection control: a paradigm shift in feedback control system design," in Proceedings of the American Control Conference (ACC '06), p. 7, IEEE, Minneapolis, Minn, USA, June 2006. 


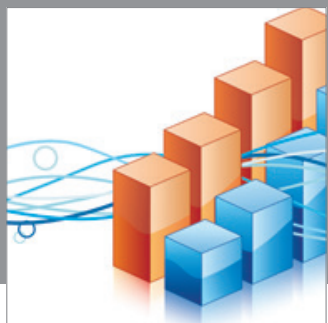

Advances in

Operations Research

mansans

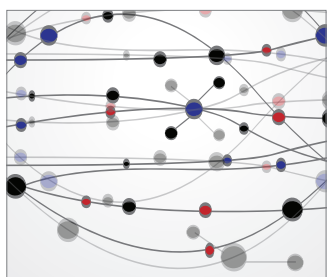

The Scientific World Journal
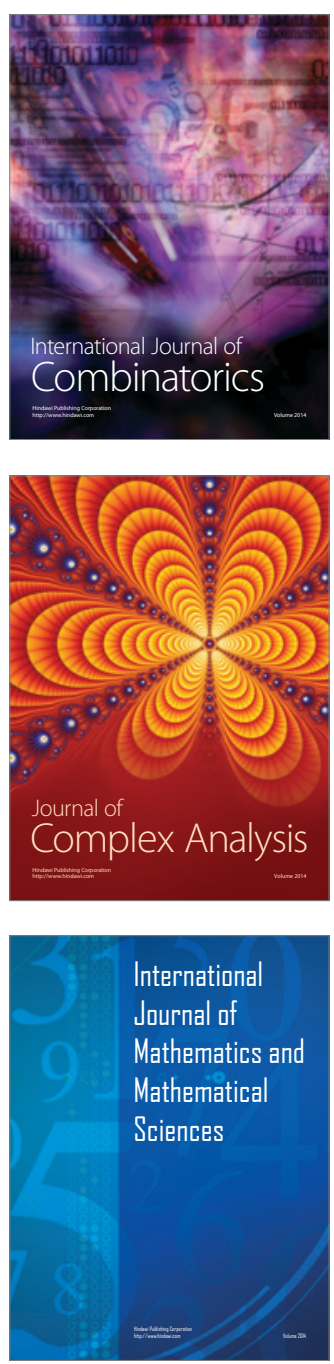
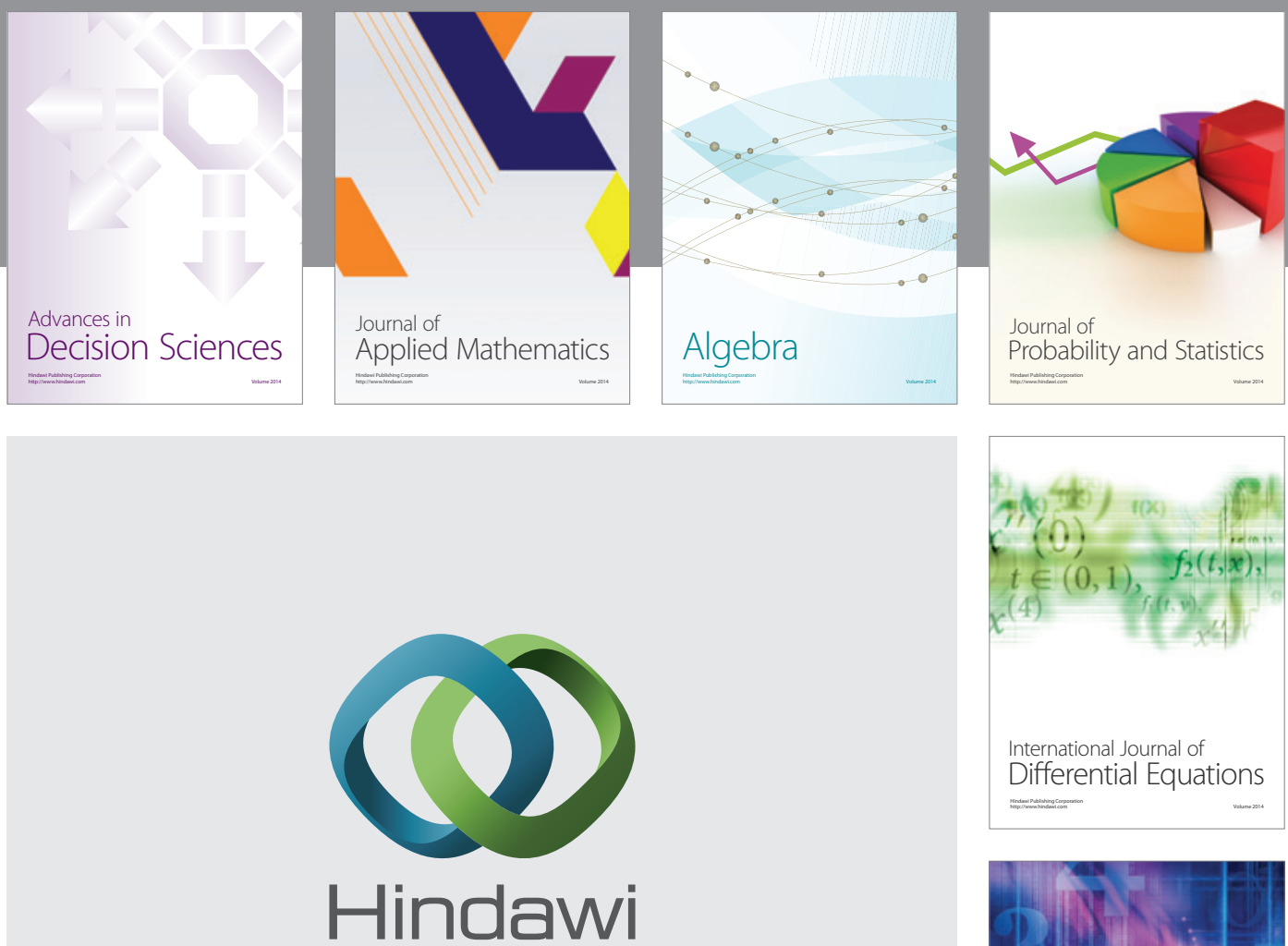

Submit your manuscripts at http://www.hindawi.com
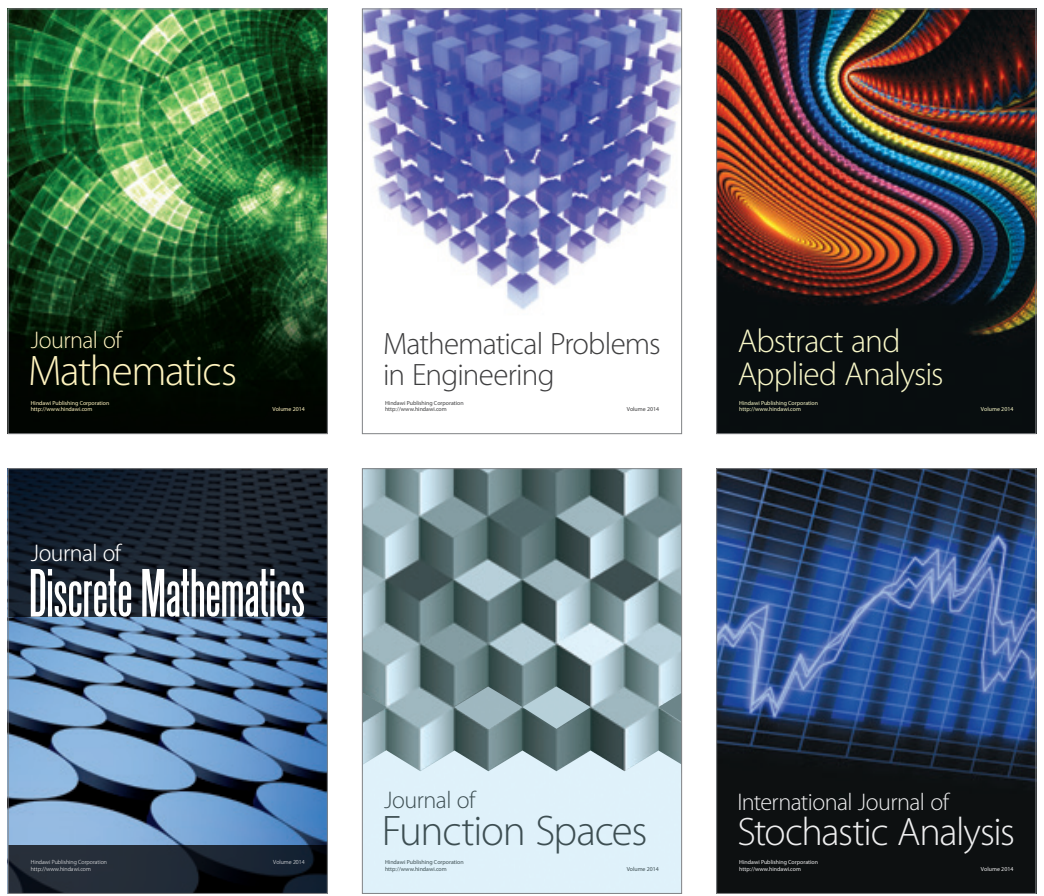

Journal of

Function Spaces

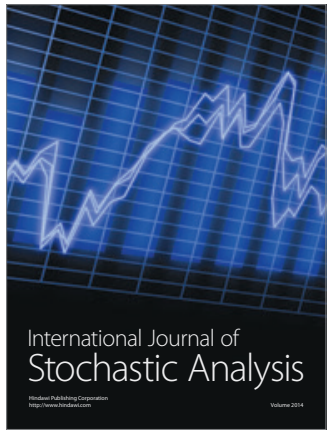

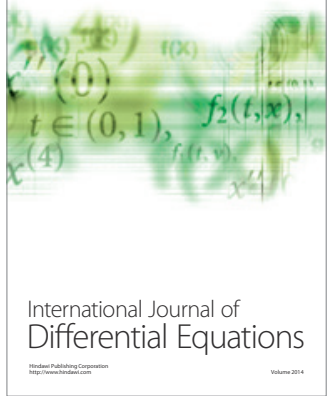
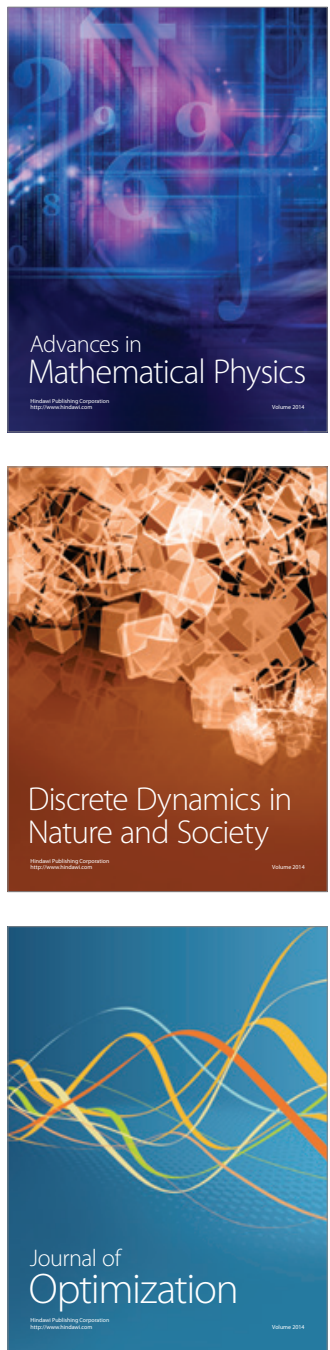